\title{
Influences of Design tools on the Original and Redesign process
}

Concept generation plays a vital role in establishing a broader foundation in the design process to create novel products. In the globalized, collaborative, designing scenario, the unambiguous representation of captured ideas to explicate the designer's thoughts is important in the sharing and reuse of concepts. Various design studies have noted the impact of design tools on concept generation. However, the results did not detail the influences of a variety of tools on the representation and reinterpretation of concepts through captured design documents. The goal of this paper is to understand the influence of conceptual design tools: Mobile e-Notes Taker ${ }^{\mathrm{TM}}$, Wacom ${ }^{\circledR}$ Tablet, and Rhinoceros ${ }^{\circledR}$ CAD with MS Word/PowerPoint on concept representation and reinterpretation, during the original and redesign phases. Eighteen design experiments, involving six individual student designers' solving three design problems each, were conducted in the original and redesign phases. The analyses of twenty six variables from captured documents and video protocols reveal that the design tools had a statistically significant impact on four key variables: the total time taken to solve each problem, the time spent on detailed design activity, the textual representation of structural requirements and the graphical representation of the structure of detailed concepts. Irrespective of the design tool used, novice designers generated a low number of redesign concepts. This makes us conclude that designers might require training for reinterpretation and extracting necessary information from the concepts originally captured, rather than working with poor understanding, ambiguity and assumptions about the original designer's intent.

Keywords: concept generation, design tools, representation, reinterpretation

\section{Introduction}

Innovation is a key factor in sustaining this competitive globalized industrial scenario. Designers are increasingly being encouraged to create quality innovative products in faster cycles. Typically, designers are trained and motivated to be creative, and creativity is often expressed through fluency, flexibility and originality (Renzulli et al., 1974). According to a common definition of creativity, "Creativity occurs through a process by which an agent uses its ability to generate ideas, solutions or products that are 
novel and valuable" (Sarkar and Chakrabarti, 2011). It has been shown that there is a positive correlation between the number and variety of ideas produced during the design process, and the novelty of the design concepts (Srinivasan and Chakrabarti, 2010a). There are many factors that influence this concept generation process. The concept generation process is currently under extensive study by the engineering community [e.g. Nagai and Taura, 2006; Srinivasan and Chakrabarti, 2010a, 2010b], with various support tools under development [e.g. Liu et al. 2000; Chakrabarti, 2001a, 2001b, 2004; Chakrabarti et al. 2005; Sarkar and Chakrabarti, 2007, 2008; Sartori et al. 2010]. Notably, people, products, processes, tools, and the organization and environment in which designing takes place (Blessing et al. 1995) have a significant impact on the concept generation process. Among these facets, design tools play a vital role in capturing designers' thought processes and in facilitating the sharing and reuse of design outcomes.

Design tools support the externalization process, which not only stores a record of design activities, but also serves as a tool to support reasoning between these sequential acts (Bilda and Gero, 2005). It has been argued that the designer should not be constrained by the tool and should be made free to express his or her intent on the design (Robertson and Radcliffe, 2009). Even though the impact of time pressure on tool selection is observed in real design practices (Elsen et al. 2010), it is a designer's responsibility to choose the appropriate design tools in the design process, based on an understanding of the ability of each tool alternative available. Stones and Cassidy (2010) argue that it is vital to equip students with the ability to make well-informed decisions about tool choice and tool use during design ideation. However, studies have shown that although CAD is less frequently used for immature designs (i.e. at the conceptual stage), it is still the most frequently used mode of working (Robertson and Radcliffe, 2009). They argue that a possible reason is the digitalization of design outcomes, which is important for future 
analysis and process integration. Ibrahim and Paulson (2008) pointed out that the transitional and iterative conceptual phase is a potential knowledge-loss period that is identified in the product development lifecycle process.

This research stems from the question whether designers really understand the influence that design tools have on the design outcomes generated. While Cham and Yang (2005) cited a number of good examples of successful integration of CAD and design education, this situation is hardly universal. The influence of design tools on creative outcomes is widely discussed in the literature. Most of the current literature has focused on original design where a designer solves a design problem for the first time. The focus of this paper is also to cover design as well as redesign processes in which the documents captured by the original designer are supplied to the re-designer (a different designer) to improve design solutions.

Ibrahim and Rahimian (2010) demonstrated that neither manual sketching tools nor CAD software are the better media for current conceptual design communications. They found that the design semantic gets lost when manual design fails in articulating an explicit design idea, while design creativity diminishes when using arduous CAD software. As the importance of the digitalization of design outcomes should be stressed along with the creativity elements, we have chosen three tools (Mobile e-Notes Taker $^{\mathrm{TM}}$, Wacom ${ }^{\circledR}$ Tablet, and Rhinoceros ${ }^{\circledR}$ CAD with MS Word/PowerPoint ) to study their impact on both original and redesign processes. These tools have the potential to capture design outcomes digitally to facilitate the redesign process.

Content-oriented studies have been carried out to study which aspects of the utilised media improve or hamper design quality. A fundamental question that this paper raises is: to what extent does the use of a particular tool impact not only the original design 
process but also the redesign process carried out by a different designer (as is typically the case)? The rest of the paper consists of sections discussing the related literature, hypotheses and methodology framed, the results obtained, and a discussion and conclusions.

\section{Related Literature}

Various design studies have been conducted to understand the differences between pencil-and-paper-aided-designing and CAD designing, especially for their impact related to creativity in design. Elsen et al. (2010) compared the pros and cons of a free-hand sketch and CAD. In this section, we summarize the results of various comparative case studies involving pencil-and-paper and CAD. Most of the studies conclude that CAD is not suitable during the conceptual stage, as it exerts a negative influence on creative design. It is commonly concluded that the creativity of designers is more effective with paper-and-pencil tools than with CAD software. The foremost reason for this conclusion is that CAD software provides inadequate $\mathrm{I} / \mathrm{O}$ systems to support intuitive idea creation (Whitefield, 1986; Kwon et al. 2003; Lawson, 2002; Stones and Cassidy, 2007). The capabilities of I/O systems are focused on the intuitive sketching capabilities offered by pencil-and-paper tools that are limited in CAD software (Kwon et al. 2005). Also the perception of visual-spatial features is high with paper-and-pencil tools (Bilda and Demirkan, 2003).

Beside inadequate I/O systems, the support offered by paper-and-pencil tools to enrich the creativity process is emphasised. Paper-and-pencil tools facilitate lateral transformations, partly due to the qualities of denseness and ambiguity found within the mark itself (Goel, 1995). Paper-and-pencil tools, through sketches, lighten the load on memory, but also support early design thinking through their ambiguity and fluidity 
(Stones and Cassidy, 2007). Also misinterpretations of the sketches could trigger novel ideas in using paper-and-pencil tools (Stacey and Eckert, 2003).

Other factors that can influence CAD suitability in the conceptual stage are the requirements for a high degree of specialisation from the users (degree of skills) (Levet et al. 2006). Designers judge their work using a different set of criteria in using different tools, e.g. a high level of finish with design proficiency in CAD software (Black, 1990). A CAD conception of the design problem is high with paper-and-pencil tools (Bilda and Demirkan, 2003). Also, CAD software uses highly structured rules leading to restriction in the early stages of design (Stones and Cassidy, 2007).

In opposition to the above conclusion, a few studies have pointed out the merits of CAD features in particular problem types. Fish and Scrivener (1990) argue that CAD software supports creativity in well-structured problems. It fosters new patterns, relationships, or aesthetics, and expands, rather than reduces the designer's creative options (Jonson, 2005). The playfulness of digital forms is evident and the fluidity of manipulation leads to new ideas (Kelly, 2001; Stones and Cassidy, 2010). Elsen et al. (2010) argue that CAD tools could be considered potentially effective also in the preliminary design process, if considered jointly with sketches.

The influencing factors generally used to assess creativity are levels of ambiguity, frequency of reinterpretation and the number and variety of concepts. Schön (1983) argues that design tools assist a 'reflective conversation' between designers and design outcomes (e.g. external representations of requirements and solutions) which help generate a mental image that, in turn, may produce more sketches (ideas) which may, again, generate another mental image, and so on and so forth (Fish and Scrivener, 1990). Stones and Cassidy (2010) define this reinterpretation as a complex, bi-directional 
cognitive process that occurs as the designer sketches. Reinterpretation is important since it is a valuable source of new, unexpected ideas, and could generally be described as the outcome of a lateral thinking process (De Bono, 1970). Goldschmidt (1994, p. 164) describes reinterpretation through stating that 'one reads off the sketch more information than was invested in its making'. It should be noted that the context of these arguments are during the phase of original design rather than the redesign process. There are contradictory opinions on the influence of tools on the reinterpretation process. Goel (1995) observed that the number of reinterpretations was higher with paper-and-pencil tools than with CAD software. The reason cited for this reinterpretation is that the designer 'sees' new ideas in the existing sketches. In opposition to the above conclusion, Won (2001) noted that the frequency of reinterpretation is higher with CAD software due to the speed of digital working i.e. the ability to 'move-see-move-see' and the facilitation of rapid transformations.

Stones and Cassidy (2010) argued that reinterpretation is linked to the quality of ambiguity. Ambiguity can be defined as 'interpretable in two or more distinct ways' or as 'vague or imprecise' (Stacey and Eckert, 2003, p. 153). They pointed out that ambiguity can lead to the discovery of useful alternative ideas, when the sketches or other communicative objects are interpreted as a different set of objects and relationships from those intended. The hypothesis is that ambiguity can be beneficial when the gain from actively clarifying shared understanding is greater than the cost of exploring unacceptable paths. Commonly, it is concluded that the level of ambiguity is higher with paper-andpencil tools than with CAD software. This is due to the visual qualities of marks in CAD which appear decisive and are notably precise, concrete and certain in finished appearance (Stones and Cassidy, 2010; Goel, 1995; Robertson and Radcliffe, 2009; Won, 2001; Lawson, 1997). 
Hannah et al. (2012) argued that designers are more confident and correct in making conclusions about whether a design meets requirements when using high-fidelity representations and physical representations, specifically high-fidelity prototypes. Lowfidelity representations (sketches and drawings) are not effective in helping to answer questions related to whether the requirements are met or not. Robertson and Radcliffe (2009) noted that the illusion of completeness offered by CAD could be a distracting factor influencing the level of ambiguity. Also, they pointed out that designers often aim for 'perfection' while using $\mathrm{CAD}$ and this influences the reinterpretation process and develops a false sense of reality.

Notably, many studies have pointed that the number of concepts is higher with paperand-pencil tools than with CAD software. Tool features such as simplicity, ease of use and ease of learning are instrumental in increasing concept generation (Stones and Cassidy, 2010; Rahimian et al. 2008). The designer's tool use also affects concept generation. Won (2001) observed that the frequent interchange between the focus on details and the focus on the whole, influences concept generation. In CAD, the switching time is much more frequent than in the traditional way. Rahimian et al. (2008) observed that maintaining the design idea during the design process, provides the ability to see all documents together and to compare them. This facilitates concept generation. Also, they pointed that ease of changing and reforming the design alternative influence this process.

As discussed in the paragraphs above, ambiguity and reinterpretation play a vital role in generating concepts. Stones and Cassidy (2010) argued that the use of symbolic systems such as fonts during synthesis tasks may restrict the scope of design ideas. Robertson and Radcliffe (2009) noted that a large amount of detail and interconnectedness is built too quickly in CAD leading to premature fixation. Premature fixation develops a resistance to change, reduces flexibility, and adversely affects motivation. Robertson et al. (2007) 
argued that the constant use of CAD under stressful conditions and circumscribed thinking interferes too strongly in the design process, either by limiting what can be created, or by encouraging the designer to over-reach the requirements of the task.

Stones and Cassidy (2010) concluded that the number of diverse concepts is higher with paper-and-pencil tools than with CAD software. They observed that CAD software is less likely to create a synthesis where shapes contribute form to each other. Also, in using CAD, designers are more likely to select a shape, rather than create it from scratch or overly manipulate it to suit the purpose (Hewson, 1994).

As predicted, the literature states that the visualization of concepts is higher with CAD software than with paper-and-pencil tools. CAD has capabilities for zooming and panning for easier walkthroughs, while temporarily omitting an object or group of objects. (Robertson et al. 2007; Robertson and Radcliffe, 2009). It also provides more detailed, realistic, and elaborated perspectives (Rahimian et al. 2008).

It is clear from these literature results, that for the conceptual stage, current CAD software is not yet a better alternative to replace conventional sketching tools, even though CAD provides enhanced visualization and speedy manipulation of objects. However, the importance of the capture and reuse of digitalized design outcomes forces us to develop enhanced novel design tools that retain the merits of both media. For developing such tools, it is vital to understand the behaviour of designers in using various advanced conceptual tools in terms of the textual and graphical representations of captured design documents. Also, the behavioural changes of designers in the reinterpretation of the captured design documents during the redesign process need to be studied across the use of various conceptual tools. The literature does not report, in any detail, the behavioural changes of designers in the representation of concepts in captured design documents. In 
the literature, various design studies have been conducted to understand the differences between pencil-and-paper-aided-designing and CAD designing, especially for their impact on creativity in design. However, substitute tools for pencil-and-paper-aideddesigning are not studied in the literature. The key gap in the literature is that the influence of a variety of tools on the representation and reinterpretation of concepts through captured design documents, is not studied. The focus of this paper is to understand the influences of three conceptual design tools - Mobile e-Notes Taker $^{\mathrm{TM}}$, Wacom ${ }^{\circledR}$ Tablet, and Computer with Rhinoceros ${ }^{\circledR}$ CAD and MS Word/PowerPoint - on concept representation and reinterpretation during the original and redesign processes. The unique feature of these three tools is their capability to digitalize design during the creation process itself. They aid designing by the capture, share and reuse of design knowledge.

\section{Research objectives and methodology}

This section provides a description of chosen design tools and the rationale for their selection, framed research hypotheses with parameter definitions, experimental design and the results of a valid coding scheme.

\subsection{Description of chosen design tools and rationale}

The aim of this paper is to study the impact of conceptual design tools on the behavioural changes of designers in (1) the representation of design concepts in design documents captured during both original and redesign processes, and (2) the reinterpretation of captured concepts during the redesign process. A concept is defined as an entity that satisfies an overall function (Srinivasan and Chakrabarti, 2010a). We have chosen Mobile e-Notes Taker ${ }^{\mathrm{TM}}$, Wacom ${ }^{\circledR}$ Tablet, and Rhinoceros ${ }^{\circledR}$ CAD with MS Word/PowerPoint (Figure 1) as the set of conceptual tools to be examined in this study. From hereafter, these three tools will be mentioned as Notes-taker, Tablet and Rhino respectively. All 
these tools are used with the help of a computer. The Notes-taker and the Tablet were selected for their potential to replace the pencil and paper tool (the most commonly used aid for conceptual design currently), and also for their ability to support the capture and reuse, in digitalized formats, of design concepts. For comparison with CAD tools, Rhino was chosen as it has been widely used in our design centre as a conceptual CAD tool. The Tablet and Rhino are widely used in design, whereas the Notes- taker is commonly used as a digital notebook for documentation purposes.

The Notes-taker is a portable handwriting capture device based on natural handwriting as an input. A plain paper of any kind can be attached to the tool and the Hi-Tech's electronic pen can be used to capture, store and share handwritten drawings, sketches and notes. In this study, we used the Wacom ${ }^{\circledR}$ DTU-710 tablet. The DTU-710 Interactive Pen display combines an LCD monitor with a tablet. This gives a direct point-and-draw-on-screen interface that can be used with a computer. Rhino is widely used during conceptual designing. Rhino offers uninhibited free-form 3-D modelling, extreme precision, unrestricted editing, 2-D drafting, annotation, illustration, compatibility, and a short learning curve. More details about these tools are provided in Appendix 1. The web links to access all appendixes of this paper are provided at the end of the references section. Table 1 summarizes the important tool-features considered in our study, which are likely to have influenced the behaviour of the designers. While using Rhino, the designers were allowed to use other word processing software such as MS Word or PowerPoint to support their designing. Since Rhino supports predominantly graphical features of designing, we provided MS word or PowerPoint to support designers in expressing textual elements. In contrast, in the Notes-taker and the Tablet, both textual and graphical elements can be represented with the same user interface. So MS word or PowerPoint was not provided while using these two tools. 


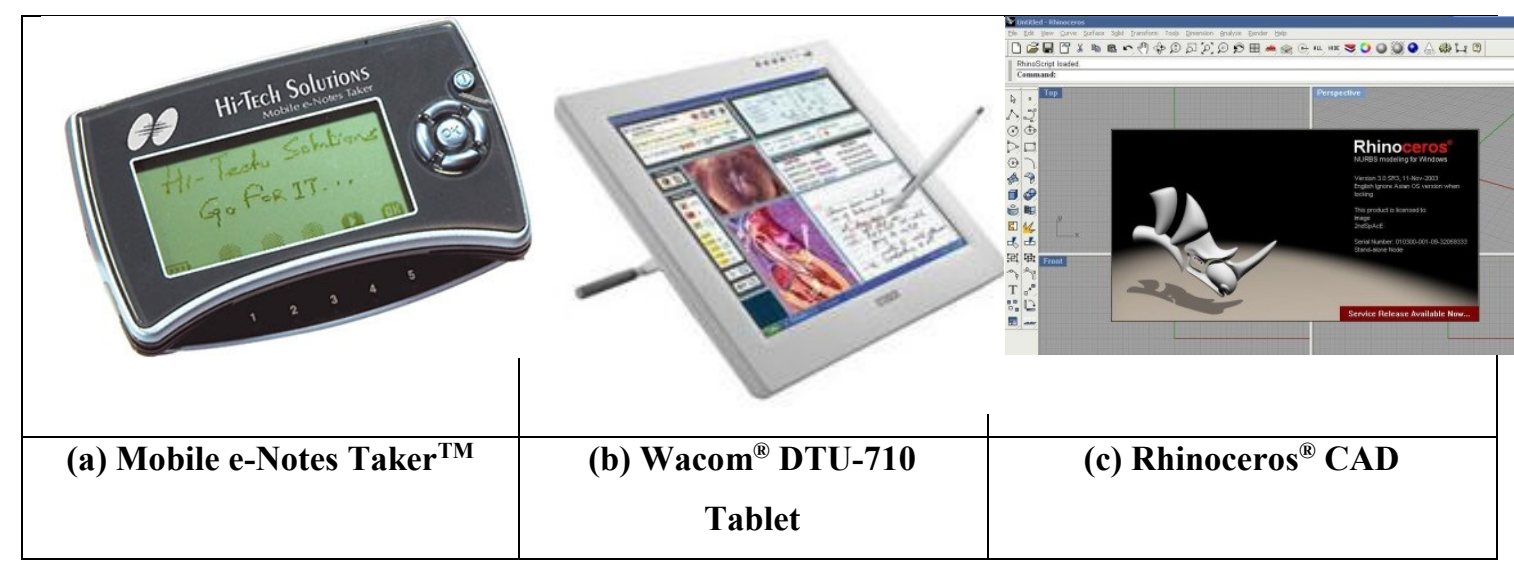

Figure 1. Conceptual design tools used in study: (a) - (c)

Table 1. Features of design tools

\begin{tabular}{|c|c|c|c|}
\hline Tool's Feature & Notes-taker & Tablet & Rhino \\
\hline Drawing medium & $\begin{array}{l}\text { A plain paper of } \\
\text { any kind and a } \\
\text { cordless Hi-Tech } \\
\text { electronic pen }\end{array}$ & $\begin{array}{l}\text { A tablet combines } \\
\text { an LCD monitor } \\
\text { (point-and-draw- } \\
\text { on-screen } \\
\text { interface) and a } \\
\text { cordless battery- } \\
\text { free pen having an } \\
\text { eraser with a } \\
\text { keyboard }\end{array}$ & $\begin{array}{l}\text { Computer screen } \\
\text { with a keyboard } \\
\text { and mouse }\end{array}$ \\
\hline $\begin{array}{l}\text { Electronic pen's } \\
\text { pressure } \\
\text { sensitivity }\end{array}$ & Not mentioned & 512 levels & Not applicable \\
\hline Coverage area & Up to A4 & 17 inch & 17 inch \\
\hline $\begin{array}{l}\text { Image resolution } \\
\text { (pixels) }\end{array}$ & $100 \mathrm{dpi}$ & $\begin{array}{l}\text { SXGA }(1280 x \\
1024)\end{array}$ & $\begin{array}{l}\text { SXGA }(1280 x \\
1024)\end{array}$ \\
\hline
\end{tabular}




\begin{tabular}{|l|l|l|l|}
\hline Tool Inclination & $90^{0}$ from vertical & Incline $18^{0}-74^{0}$ & $0^{0}$ from vertical \\
(degrees) & & from vertical & \\
\hline
\end{tabular}

\subsection{Parameter Definition and Research Hypotheses}

A map of the set of hypotheses explored in this work is shown in Figure 2. The parameters used for the investigation are identified and these are the 'Number of preliminary and detailed concepts', 'time taken across the design activities', 'representation of \{requirements, preliminary ideas and detailed concepts\}', and 'reinterpretation of captured documents'. In addition to the four parameters detailed in Figure 2, 'designer adaptability to design tool' is also studied. The effect of all these five parameters is studied in detail using the procedure discussed in subsequent paragraphs. Representation is studied through textual and graphical formats. Textual contents are analysed by counting the number of words used to express function, behaviour and structure elements; whereas graphical contents are analysed using the number of distinguishable components represented through sketches and diagrams. For the distinguishing function, behaviour and structure elements, the definitions used by Chakrabarti et al. (2005) are used.

- Function: Descriptions of what a system does: it is intentional and generally at a higher level of abstraction than behaviour.

- Behaviour: Descriptions of how a system performs its function. This is generally at a lower level of abstraction than function.

- Structure: Structure is described by the elements and interfaces with which the system and its immediate interacting environment are constructed.

The reinterpretation of captured concepts is analysed by the understanding, ambiguity, assumptions and repetitions made by the designer working in the redesign phase. Ambiguity can be defined as 'interpretable in two or more distinct ways' or as 'vague or 
imprecise' (Stacey and Eckert, 2003). Video protocols have been analysed to segment the ambiguous portions expressed by each designer. Adaptability with design tools has been studied through the comfort of the designer. Video protocols and audio transcripts have been used to understand and segment portions of uncomfortable behaviours. The time taken across the design activities is noted by using timestamps in the video protocols. The formulated hypotheses which are verified in this study are given below:

(H1) Conceptual design tools have a significant impact on the number of concepts generated.

(H2) Conceptual design tools have a significant impact on the amount of time spent by the designers across design stages in both the original and redesign phases.

(H3) Conceptual design tools have a significant impact on the representation (wider variation possible in the expression of design elements through textual (the number of words) and graphical (distinguishable components) format) features of captured requirements and concepts in terms of functional, behavioural and structural elements.

(H4) The amount of time taken to capture requirements and concepts has a significant impact on the representation of captured documents.

(H5) Formats of the representation of captured documents have a significant impact on reinterpretation in the redesign phase.

(H6) Designer adaptability to a design tool has a significant impact on the representation and reinterpretation of captured documents.

The significant term in these hypotheses represents the statistical significance of the results. Since the results represented in this work are from the descriptive studies, discussed above, the data has been analyzed without any expectations. In doing so, the authors' bias has been reduced. 


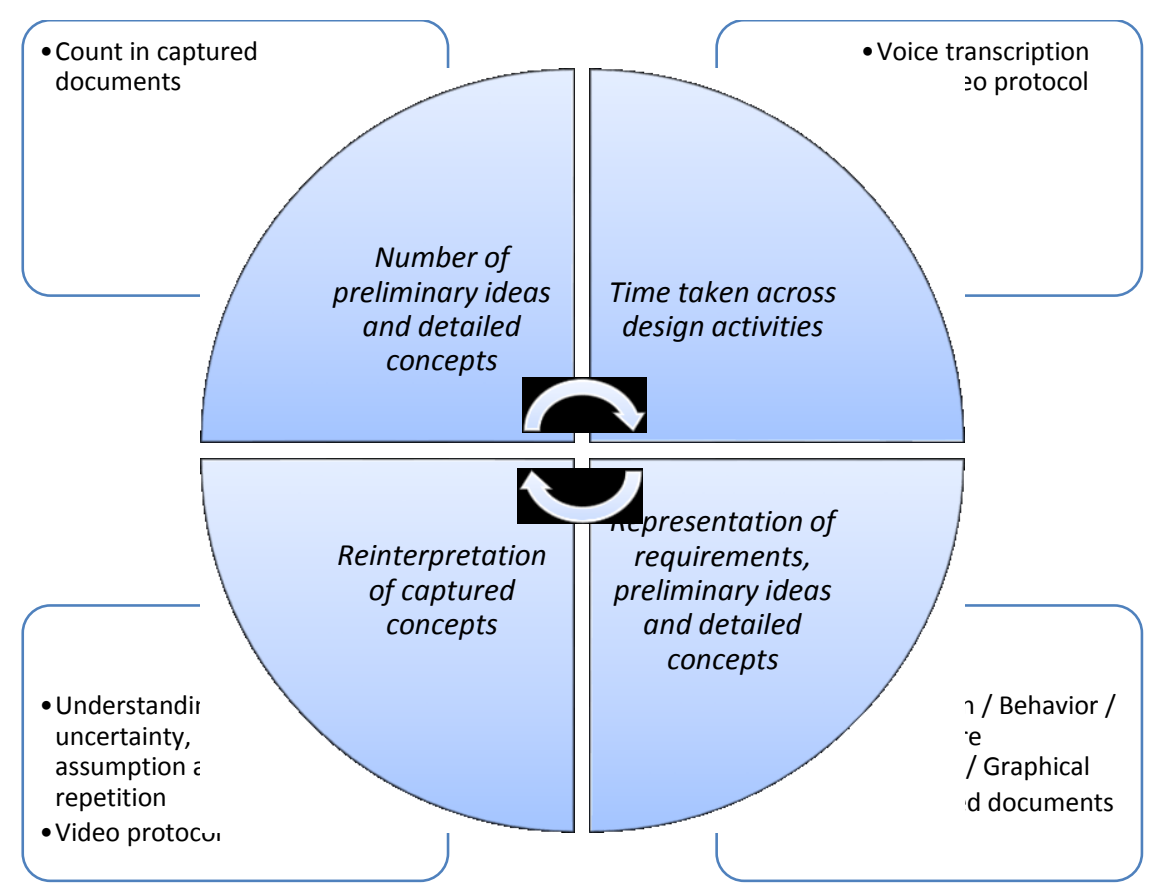

Figure 2. Research hypotheses map

\subsection{Design of Experiment}

To verify the formulated hypotheses, in-house experiments were conducted in a laboratory setting. To study the capture and reuse aspects, original and redesign experiments were conducted in a systematic manner. The experiments were structured taking into account the variation necessary to enable a comparison of the use of the three design tools, by multiple people, in both the capture and reuse aspects. The present problem involves three principal effects: design tools, design problems and designers. The experimental treatment combinations are generated by taking the help of the design of experiments approach. The design of experiments approach helps in reducing the number of experiments to be conducted to test the significance of the identified hypotheses. The factors used for the experiments are fixed. The levels are three, six and six for design tools, design problems and designers respectively. A constrained 6 (designer) x 6 (design problems) Latin square is shown in Table 2. It should be noted in Table 2 that every (numbered) level of the factor of interest appears once in each row and 
column of the table. Three different original design problems and three corresponding redesign problems were used through the 18 experiments. The main aim for conducting each of the nine experiments in the original and redesign process was to overcome and understand the variation possible in solving different design problems through different tools. It was not aimed at finding an equivalent problem in each set. The constraints applied while implementing this 6 x 6 Latin square design are discussed below.

- A designer should NOT solve the same problem with different tools (repetition is not allowed).

- Original and redesign problems (e.g., P1, P1') should not be solved by the same designer.

- Each designer should solve three different design problems, each with a different tool.

- In order to maximize the randomization of the designer's problem assignment, it is suggested that each designer should solve at least one original design problem or one redesign problem. That is, each designer will get either two original and one redesign problem, or one original and two redesign problems.

Due to these constraints, the experiments mentioned in the crossed three rows in Table 2 were not conducted. The analyses were based only on the results derived from the first three rows. The response variable under study is represented using the basic statistical model for the Latin square design:

$y_{i j k}=\mu+\alpha_{i}+\beta_{j}+\gamma_{k}+\epsilon_{i j k}\{$ where $i=1,2,3 ; j=1,2 . .6 ; k=1,2, . .6$

Where $y_{i j k}$ is the response measure for observation in a set of a ' $i$ ' design tool, a ' $j$ ' design problem and a ' $k$ ' designer; $\mu$ denotes the population mean, $\alpha_{i}$ the effect of the 
design tool $i, \beta_{j}$ the effect of the design problem $j, \gamma_{k}$ the effect of the designer $k$ and $\epsilon_{i j k}$ the error factor. Assuming simple additivity of the effect of design tools, design problems and designers is a good approximation for the small range of observations, and this design permits an independent and unbiased estimation of all the three effects. $\left(\alpha_{i}\right.$, $\left.\beta_{j}, \gamma_{k}\right) \sim N\left(0, \sigma_{i j k}^{2}\right)$ is a nominal offset for set $N\left(\theta, \sigma^{2}\right)$ denoting 'distributed as a normal distribution with mean $\theta$ and variance $\sigma^{2}$. The extraction of the main effects and relevant variance components from a Latin square design is derived using general linear modelling functions in Minitab $^{\circledR}$ (version 15.1) software. The level of significance considered in all hypothesis testing is 0.05 .

Table 2. Structure of the design of experiments (D1 - D6: Designers, P1 - P3: Original problem; P1' - P3': Redesign problem) (crossed experiments were not conducted due to experimental constraints)

\begin{tabular}{|c|c|}
\hline Tools & Design problem \\
\hline
\end{tabular}




\begin{tabular}{|c|c|c|c|c|c|c|}
\hline \multirow{2}{*}{ Tablet } & \multicolumn{3}{|c|}{ Original } & \multicolumn{3}{c|}{ Redesign } \\
\cline { 2 - 6 } Rhino with MS & P1 & P2 & P3 & P1' & P2' & P3' \\
Word/PowerPoint & & D3 & D4 & D5 & D2 & D6 \\
\hline Notes-taker & D6 & D1 & D3 & D4 & D5 & D1 \\
\hline Thino with MS & D5 & & & & & \\
\hline
\end{tabular}

Overall, 18 experiments were conducted with four Master of Design students and two design researchers. These designers are involved in nine experiments each involving original design and redesign. These eighteen experiments were conducted because,

- It was planned to conduct more than one experiment in each set involving an original or redesign experiment using a specific tool (e.g., Notes-taker and Original) because the results are better validated with multiple experiments each involving different problems and designers using the same tool.

- Conducting two experiments in each set does not provide symmetry to the design of experiments because the number of tools considered for this study is three. This would result in a designer solving the same problem twice using different tools. This would create problems while carrying out comparisons for the same tool across original and redesign experiments. Hence three experiments in each set were carried out. 
- Also, three experiments in each set provide a confirmation of the results produced in these analyses.

- Since the three experiments in each set provide symmetry to the designed experiments, three different design problems were framed for the original, and three corresponding ones for the redesign experiments. Six designers participated in these experiments. Each designer solved three different problems using three different tools. This set-up tally with the symmetry developed in the design of experiments.

All the captured original design documents (in the same captured format) were given to the re-designer (designer involved in redesign) along with the redesign problem. The re-designer was given freedom to explore and reinterpret the original design documents at his leisure. Details about the participants/subjects who participated in these experiments are given in Table 3 . The time limit mentioned in the problem sheet was approximately one hour. The reasons for this approximate time are:

- Designers wanted to solve the given problem completely to their satisfaction.

- Forcing designers to work compulsorily for 1 hour might have changed their behaviour significantly.

The challenge is to allow designers to work as naturally as possible while enabling the use of the tool. The three design problems and their corresponding three redesign problems are described in Appendix 2. The designers were given adequate training to use the tool before conducting the experiments. Every experiment was started after getting the 'go-ahead' signal from the participating designer, when he felt competent to solve the problem with the given tool. However, their competence levels were not judged before each experiment. During the designed experiment, each subject was asked to 'think aloud' such that the researcher could obtain a rich externalization of their thoughts and activities 
from the experiments. It should be noted that to nullify the effects of the 'sequence in which tools were used', the experiments were conducted in a randomized fashion. All the experiments were video recorded. Voice protocols, captured documents and video recordings provided the material to test the hypotheses framed.

Table 3. Details about the subjects who participated

\begin{tabular}{|c|c|c|c|c|}
\hline Designers & Current studies & $\begin{array}{l}\text { Educational } \\
\text { background }\end{array}$ & $\begin{array}{l}\text { Years of } \\
\text { industrial } \\
\text { experience }\end{array}$ & $\begin{array}{l}\text { Number of } \\
\text { products } \\
\text { designed }\end{array}$ \\
\hline D1 & $\begin{array}{c}1^{\text {st }} \text { year student- Master } \\
\text { of Design (M.Des) }\end{array}$ & B.E. Mechanical & 2.5 & $\sim 5$ \\
\hline $\mathrm{D} 2$ & $1^{\text {st }}$ year student - M.Des. & $\begin{array}{l}\text { B.Tech. } \\
\text { Electronics and } \\
\text { Communication }\end{array}$ & 0 & $\sim 3$ \\
\hline D3 & $2^{\text {nd }}$ year student - M.Des & B.E. Production & 1 & $\sim 10$ \\
\hline D4 & $\begin{array}{c}\text { Research Fellow - Ph.D. } \\
\text { Completed }\end{array}$ & M.Des. & 2.5 & $\sim 10$ \\
\hline D5 & $4^{\text {th }}$ year student - Ph.D. & $\begin{array}{l}\text { M.Tech. } \\
\text { Mechanical }\end{array}$ & 0 & 0 \\
\hline D6 & $\mathrm{I}^{\text {st }}$ year student - M.Des. & B.E. Mechanical & 2 & $\sim 4$ \\
\hline
\end{tabular}

\subsection{Coding scheme validation}

The lead researcher of this paper carried out all of the categorisation of the data using the parameter definitions and documented procedure set out in Appendix 3. A complete experiment was categorized by two engineers working together using Appendix 3 as guidance. Since the two engineers were new to the research coding process, both were 
trained for two weeks using other coding materials. The transcripts of the coder for the two experiments were compared to that of the researcher. Appendix 3 documents a sample of the reported transcript. The overall agreement percentage is $96 \%$. The disagreements which took place in misinterpretation between function and behaviour arose because of a misunderstanding of the context, negligence in choosing all the captured words, difficulties in splitting function, behaviour and structure in a sentence, differentiating text and graphical elements separately, merging of two concepts due to improper labelling, and the exclusion of dimensions in structural elements. Appendix -3 provides clarification for the feedback received in this testing process.

\section{Results}

H1. Conceptual design tools have a significant impact on the number of concepts generated.

Tables 4 and 5 give the number of preliminary and detailed concepts generated in the original and redesign experiments across the three design tools. The captured documents are analysed to count the number of captured concepts. A preliminary concept is defined as an idea to solve a given design problem, whereas a detailed concept is taken as one that is elaborated. Figure 3 illustrates an example of a preliminary and detailed concept. Designers using Rhino chose MS PowerPoint or Word to explore preliminary concepts, and Rhino to elaborate and detail the design. To identify the statistical significance of the effect of design tools, design problems and designers on the number of concepts generated, we have used general linear modelling (GLM) in an Analysis of Variance (ANOVA) (discussed in Section 3.3). The results reveal that the influence of design tools on the preliminary concept $(\mathrm{F}(2,5)=0.50, \mathrm{p}=0.636)$ and detailed concept $(\mathrm{F}(2,5)=4$, $p=0.092)$ is statistically insignificant. Since the focus of this paper is primarily on the 
design tools, the impact of the design problem and the designer is not discussed unless any significance is noted in the statistical analyses. However, the complete results along with the data sets are documented and presented in Appendix 4.

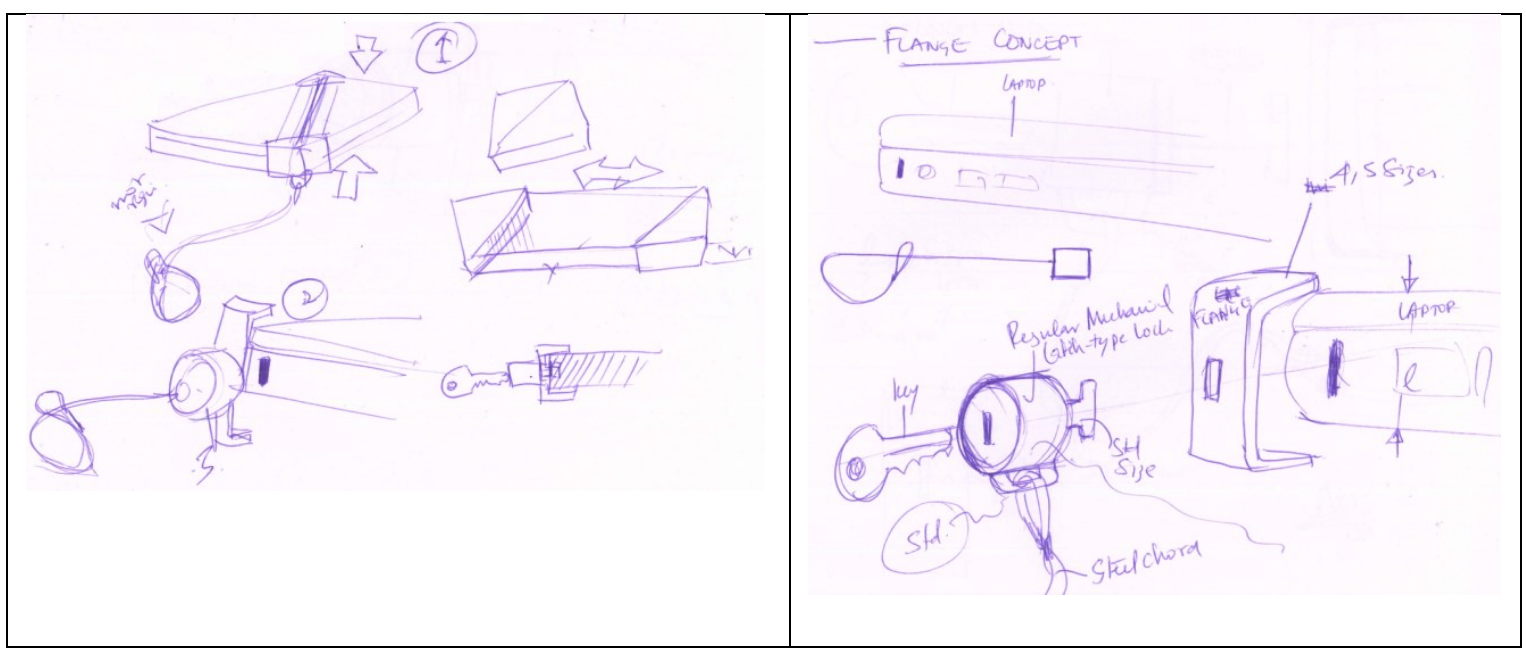

Figure 3. An example of preliminary and detailed concepts generated for laptop security issues

Table 4. Number of preliminary concepts generated in the original and redesign experiments

\begin{tabular}{|c|l|l|l|l|l|l|}
\hline Tools & P1 & P2 & P3 & P1' & P2' & P3' \\
\hline Tablet & 0 & 1 & 1 & $\mathbf{1}$ & $\mathbf{0}$ & $\mathbf{0}$ \\
\hline Rhino with MS & 0 & $2(2)$ & 10 & $\mathbf{0}$ & $\mathbf{0}$ & $\mathbf{1}(\mathbf{1})$ \\
Word/PowerPoint (number & & & $(10)$ & & & \\
\hline
\end{tabular}




\begin{tabular}{|c|c|c|c|c|c|c|}
\hline $\begin{array}{c}\text { of concept generated in MS } \\
\text { Word/PowerPoint in } \\
\text { bracket) }\end{array}$ & & & & & & \\
\hline Notes-taker & 7 & 7 & 0 & $\mathbf{1}$ & $\mathbf{0}$ & $\mathbf{0}$ \\
\hline
\end{tabular}

Even though the influence of design tools on concepts generation is insignificant, in Table 4, we derived two critical observations (bold numbers): (1) The number of preliminary concepts generated through the Tablet tool is substantially lower than with the other two tools and (2) Irrespective of the design tools, the number of preliminary concepts generated in the redesign process is substantially lower. The reason for the fewer number of preliminary concepts in the Tablet tool could be the designers' intention to detail every concept in the Tablet tool. This behaviour could be due to the 'point-and-draw-on-screen' interface provided by the Tablet tool which might have led to design fixation. This behavioural change could have had a severe impact on the design outcomes, based on the argument of Srinivasan and Chakrabarti (2010) that variety in the concepts increases the novelty of the design outcomes. We will discuss the redesign outcomes along with the factors discussed in the following hypotheses.

Table 5. Number of detailed concepts generated in the original and redesign experiments

\begin{tabular}{|l|c|l|l|l|l|l|}
\hline Tools & P1 & P2 & P3 & P1' & P2 & P3' \\
& & & & & & \\
\hline Tablet & 5 & 3 & 2 & 1 & 3 & 1 \\
\hline Rhino with MS & $1(1)$ & $3(3)$ & $1(1)$ & $1(1)$ & 2 & $1(1)$ \\
Word/PowerPoi & Descriptio & Descriptio & Descriptio & Descriptio & $(0)$ & Descriptio \\
\hline
\end{tabular}




\begin{tabular}{|c|c|c|c|c|c|c|}
\hline nt (number of & n shared & n shared & n shared & n shared & & n shared \\
concept & in both & in both & in both & in both & & in both \\
generated / & tools & tools for & tools & tools & & tools \\
shared in MS & & one & & & & \\
Word/PowerPoi & & concept & & & & \\
nt in bracket) & & & & & & \\
\hline Notes-taker & 4 & 2 & 2 & 2 & 1 & 2 \\
\hline
\end{tabular}

For the detailed concepts, it is good to note that the Tablet tool dominates the other tools in the number of detailed concepts, since producing fewer concepts both in the preliminary and detailed concepts might have a significant impact on the design outcomes. The number of detailed concepts in Rhino is lower compared to the other tools. This trend could be due to the individual or combined influences of premature fixation, as pointed out by Robertson et al. (2007), the one hour approximate experiment timing, and the designers' skills in using Rhino software. However, it has been observed that using other word processing software such as MS PowerPoint or MS Word along with Rhino reduced the influence of these factors. The total number of concepts generated in the Notes-taker is substantially higher than when using other tools. This trend could be due to the natural paper and pencil interface which provides fluidity in designing without any limitations from software features. Overall, the statistical results indicate that the three conceptual design tools chosen do not have a significant impact on the number of design concepts generated. So the first hypothesis (H1) is rejected.

\section{H2. Conceptual design tools have a significant impact on the amount of time spent} by the designer across design stages.

Table 6 provides the total amount of time taken in each experiment by the designers using 
the three different tools, in the original and redesign experiments. The overall time spent with Rhino with MS Word/PowerPoint is much higher than with the other tools. It is interesting to see the variation of the overall time taken. The Notes-taker tool has both extremes of minimum and maximum time taken. The trend of overall reduction in the time taken in the redesign, rather than the original experiments, is observed clearly with the Notes-taker tool. Figures 4 and 5 show the distribution of time taken across the design stages combining all original and redesign experiments, respectively. Video protocols have been used to segment and record the amount of time spent on each design stage. From Figures 4 and 5 it can be inferred that the Notes-taker tool had took the maximum time in the original design process due to the significant amount of time spent on the requirement generation process compared to the other two tools. The Notes-taker tool got very little time in the redesign process because of less time spent on requirement generation and detailing processes on the concept chosen.

Table 6. Total time taken by the designers in original and redesign experiments (in

\begin{tabular}{|c|c|c|c|c|c|c|c|c|c|c|c|}
\hline Tools & P1 & $\mathbf{P 2}$ & P3 & $\begin{array}{c}\text { P1 } \\
,\end{array}$ & $\begin{array}{c}\mathbf{P 2} \\
,\end{array}$ & $\begin{array}{c}\text { P3 } \\
\text {, }\end{array}$ & $\begin{array}{c}\text { Mi } \\
\text { n }\end{array}$ & $\begin{array}{c}\text { Averag } \\
\text { e } \\
\text { origina } \\
\text { I time }\end{array}$ & $\begin{array}{l}\text { Averag } \\
\text { e } \\
\text { redesig } \\
\text { n time }\end{array}$ & $\begin{array}{c}\mathbf{M a} \\
\mathbf{x}\end{array}$ & $\begin{array}{c}\text { Tot } \\
\text { al }\end{array}$ \\
\hline Tablet & 34 & 66 & 53 & 25 & 64 & 87 & 25 & 51 & 58.7 & 87 & 329 \\
\hline
\end{tabular}




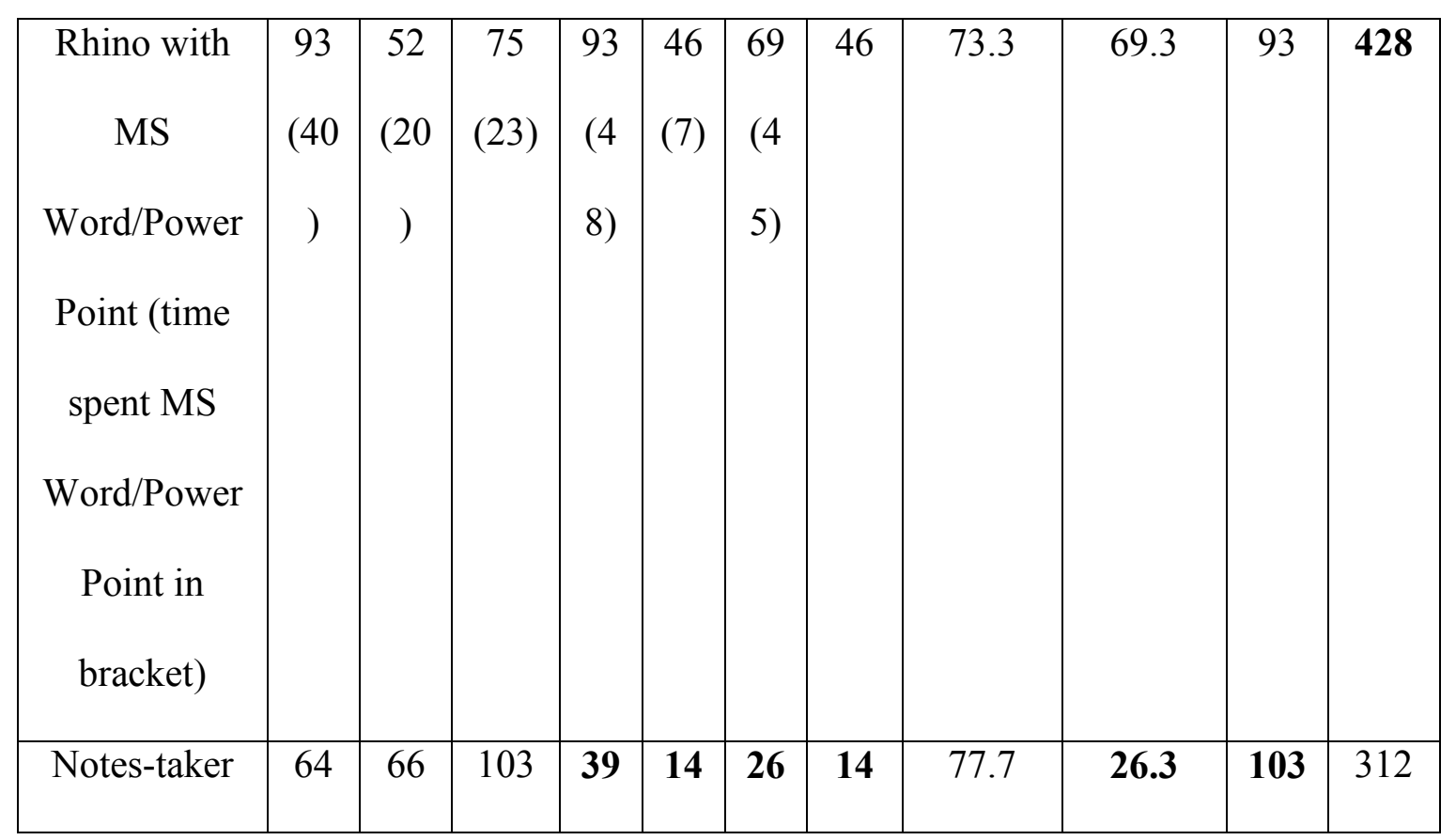

In the original experiments, the distribution for Rhino with MS Word/PowerPoint varied significantly as compared to the other two tools, especially in the 'conceptual elaboration' and 'detailing the concept chosen' stages. The fixation highlighted in the previous hypothesis in using Rhino is indicated by the amount of time spent on detailing chosen concepts. However, in the redesign experiments, the difference between the amount of time spent in the 'conceptual elaboration' and 'detailing chosen concept' stages is substantially reduced $(10 \%$ compared to $32 \%)$ in using Rhino with MS Word/PowerPoint.

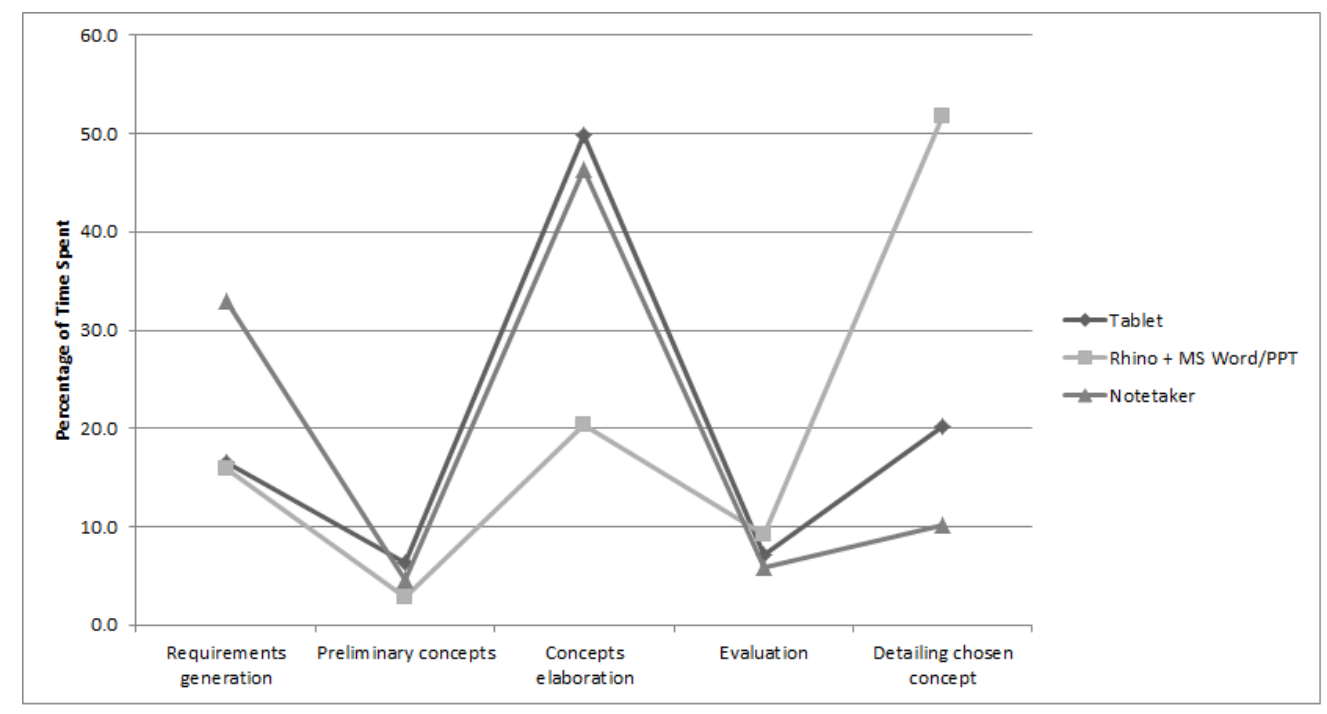


Figure 4. Distribution of time spent on design activities in original experiments (all nine combined)

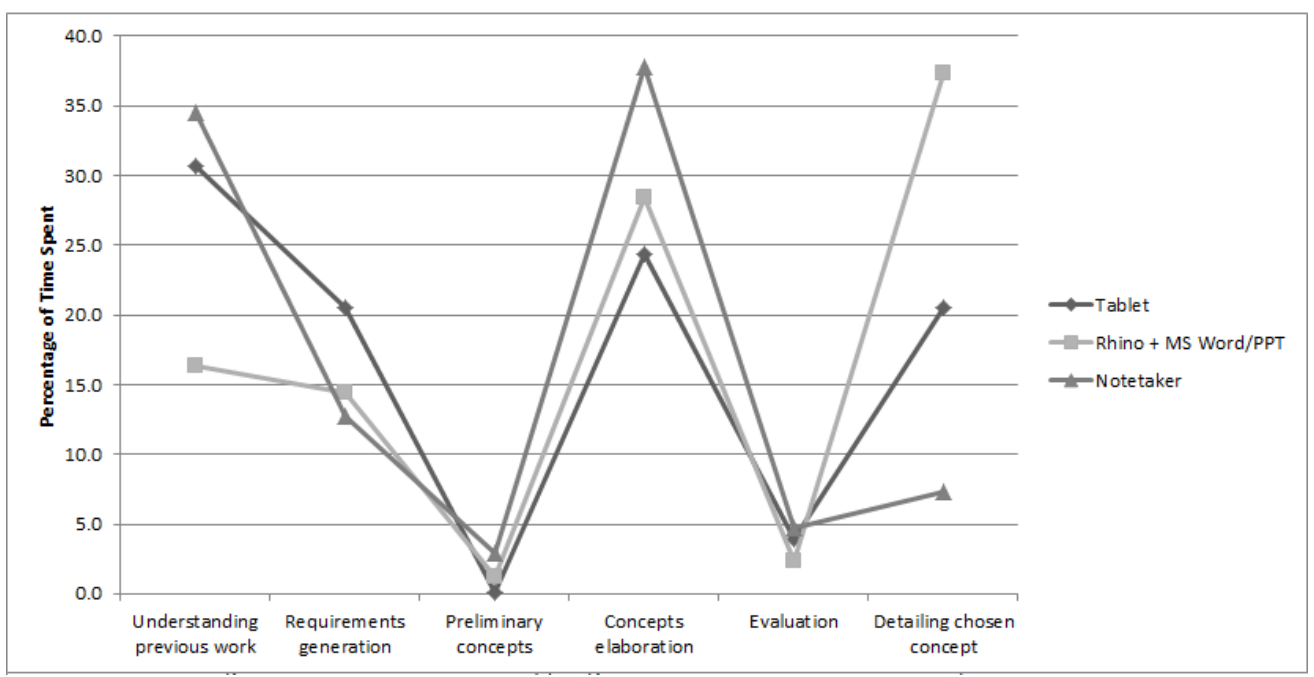

Figure 5. Distribution of time spent on design activities in redesign experiments (all nine combined)

With the available data, ANOVA is carried out using GLM. The results revealed statistically significant differences among the design tools and designers in relation to the total time taken to solve design problems $\left(\mathrm{F}_{\text {Design_tool }}(2,5)=6.85, \mathrm{p}=0.037 ; \mathrm{F}_{\text {Designer }}(5\right.$, $5)=15.02, p=0.005)$. A very high $R^{2}$ value $(R-S q=95.69 \%)$ indicates how well the model predicts responses. Figure 6 represents the mean plot for the total time with reference to the design tool, design problem and designer. Compared to the other tools, the total time taken in Rhino with MS Word/PowerPoint is high.

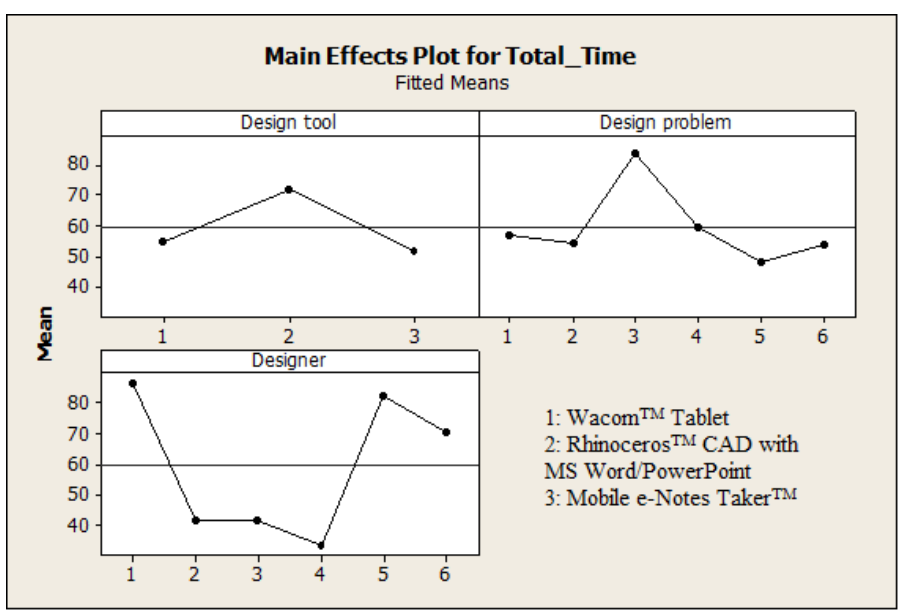


Figure 6. Mean plot for total time with reference to design tool, design problem and designer

The test results revealed statistically insignificant differences among the design tools in relation to the time spent on requirement generation activity $(F(2,5)=0.56, p=0.603)$. However, the influence of the designer on the time spent on requirement generation activity is statistically significant $(\mathrm{F}(5,5)=7.38, \mathrm{p}=0.023)$. Out of 18 experiments, in nine experiments, the designers did not spend much time on preliminary idea generation activity. So, no influence of design tools on preliminary idea generation is observed. Also, the influence of the design tools on the time spent in concept elaboration and evaluation activity are statistically insignificant $\left(\mathrm{F}_{\text {concept_elaboration }}(2,5)=0.17, \mathrm{p}=0.849\right) ;\left(\mathrm{F}_{\text {Evaluation }}\right.$ $(2,5)=0.38, p=0.699)$. The ANOVA test results revealed statistically significant differences among the design tools in relation to the time spent on detailed design activity $(\mathrm{F}(2,5)=5.93, \mathrm{p}=0.048)$.

In redesign experiments, the designers spent an average of $30-35 \%$ of the time, while using the Notes-taker and the Tablet tools in understanding, evaluating and selecting previous designs. This shows that the designers are keen to understand previous designs from the captured documents. However, with Rhino with MS Word/PowerPoint, the designers on average spent only $16 \%$ of the time in understanding previous work. The reasons for this time variation are discussed along with the design representation elaborated in the next hypothesis. The significant amount of time spent in the 'concept elaboration' stage with Notes-taker could be one reason for the higher number of concepts generated, as explained in the previous hypothesis. These results indicate that conceptual design tools have a significant impact on the total time spent by the designers on solving design problems and the time spent in the detailed design activity. Also, the influence of the designer on the total time taken to solve design problems and the time spent in 
requirement generation activity are identified.

It is notable that the percentage of time spent by the designers in the 'preliminary concept' stage is low and did not vary considerably across different tools. Further research is required to explore this phase in detail in order to understand the impact of this stage on design outcomes.

H3. Conceptual design tools have a significant impact on the representation (graphical and textual format) of captured requirements and concepts in terms of functional, behavioural and structural elements.

H4. The amount of time taken to capture requirements and concepts has a significant impact on the representation of captured documents.

Captured documents were analysed to segregate the number of words and distinguishable components used to represent concepts. Figure 7 illustrates the textual and graphical classification into function, behaviour and structure elements. It should be noted that the words and diagrams were analysed as they were, and no further interpretation was carried out in the analyses. Table 7 shows the textual and graphical content in terms of functional, behavioural and structural elements in the captured requirements, in both the original and redesign experiments. 


\begin{tabular}{|c|c|c|}
\hline . & 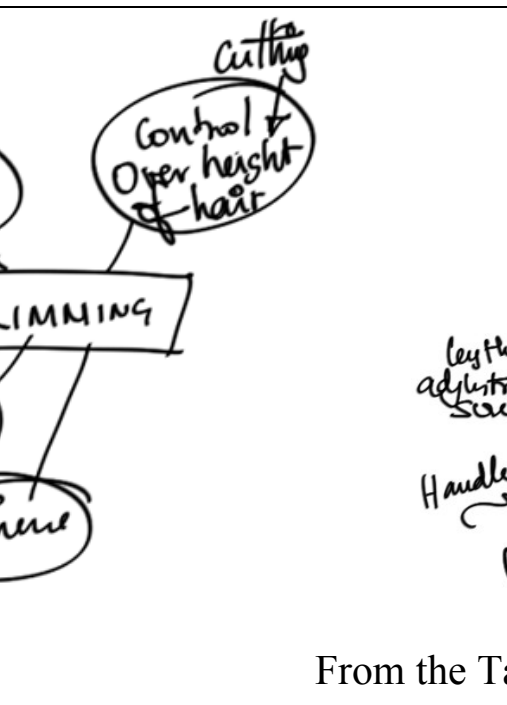 & adjustmant \\
\hline & $\begin{array}{l}\text { Textual (number of words } \\
\text { in bracket) }\end{array}$ & $\begin{array}{l}\text { Graphical (number of } \\
\text { components in bracket) }\end{array}$ \\
\hline Function & $\begin{array}{l}\text { Trimming - less time, safety, } \\
\text { hygiene, control over cutting } \\
\text { height of hair (11) }\end{array}$ & - \\
\hline Behaviour & $\begin{array}{l}\text { Adjustment knob; length } \\
\text { adjustment screw; } \\
\text { Adjustment (6) }\end{array}$ & - \\
\hline Structure & Razors; handle (2) & $\begin{array}{l}\text { Handle, handle grip, two screws, } \\
\text { razors, knob, supporting structure, } \\
\text { comb (8) }\end{array}$ \\
\hline
\end{tabular}

Figure 7. Illustration of the textual and graphical classification into function, behaviour and structure elements

Table 7. Representation formats of captured requirements combining all original and redesign experiments (Function/Behaviour/Structure) 


\begin{tabular}{|c|c|c|c|c|c|c|c|c|c|c|c|c|}
\hline \multirow[t]{3}{*}{ Tools } & \multicolumn{6}{|c|}{ Original } & \multicolumn{6}{|c|}{ Redesign } \\
\hline & \multicolumn{3}{|c|}{ Textual } & \multicolumn{3}{|c|}{ Graphical } & \multicolumn{3}{|c|}{ Textual } & \multicolumn{3}{|c|}{ Graphical } \\
\hline & Fun. & Beh. & Str. & Fun. & Beh. & Str. & Fun. & Beh. & Str. & Fun. & Beh. & Str. \\
\hline Tablet & 80 & 36 & 31 & 0 & 0 & 0 & 85 & 48 & 8 & 0 & 0 & 0 \\
\hline Rhino with & 43 & 32 & 80 & 0 & $2(2)$ & 5 & 48 & 59 & 95 & 0 & 0 & 0 \\
\hline MS & (43) & (32) & $(80$ & & & (5) & (48) & (59) & (95 & & & \\
\hline Word/Pow & & & ) & & & & & & ) & & & \\
\hline erPoint & & & & & & & & & & & & \\
\hline (MS & & & & & & & & & & & & \\
\hline Word/Pow & & & & & & & & & & & & \\
\hline erPoint & & & & & & & & & & & & \\
\hline representat & & & & & & & & & & & & \\
\hline ion is & & & & & & & & & & & & \\
\hline given in & & & & & & & & & & & & \\
\hline bracket) & & & & & & & & & & & & \\
\hline Notes- & 394 & 55 & 78 & 1 & 0 & 24 & 46 & 0 & 4 & 0 & 0 & 1 \\
\hline taker & & & & & & & & & & & & \\
\hline
\end{tabular}

The influence of the design tools on the textual representation of functional and behavioural requirements is statistically insignificant $\left(\mathrm{F}_{\text {textual_function }}(2,5)=1.97, \mathrm{p}=\right.$ $\left.0.234 ; F_{\text {textual_behavioural }}(2,5)=1.65, \mathrm{p}=0.282\right)$. However, the influence of the designer on the textual representation of behavioural requirements is statistically significant $\left(F_{\text {textual_behavioural }}(5,5)=15.19, \mathrm{p}=0.005\right)$. The influence of the design tools on the textual representation of structural requirements is statistically significant $\left(F_{\text {textual_structure }}(2,5)=\right.$ $6.30, \mathrm{p}=0.043$ ). No statistical influence of the design tools on the representation of graphical requirements is identified because none of the designers significantly represent 
the requirements graphically in any of these three tools. The observations from Table 7 are the following:

- Overall, textual descriptions dominate, in representing problem analyses and requirements, for all three tools.

- The textual descriptions of functional requirements in the original experiments are substantially higher in the Notes-taker than in the other tools. This could be due to the substantial amount of time spent (about 32\%) using this tool at the 'requirements generation' stage (Figure 4).

- In the original experiments with the Notes-taker tool, graphical elements are used primarily to represent the requirements of structural elements.

Table 8 shows the number of textual and graphical contents in terms of the functional, behavioural and structural elements of the captured preliminary concepts in the original and redesign experiments.

Table 8. Representation formats of the captured preliminary concepts in the original and redesign experiments (Function/Behaviour/Structure) 


\begin{tabular}{|c|c|c|c|c|c|c|c|c|c|c|c|c|}
\hline \multirow[t]{3}{*}{ Tools } & \multicolumn{6}{|c|}{ Original } & \multicolumn{6}{|c|}{ Redesign } \\
\hline & \multicolumn{3}{|c|}{ Textual } & \multicolumn{3}{|c|}{ Graphical } & \multicolumn{3}{|c|}{ Textual } & \multicolumn{3}{|c|}{ Graphical } \\
\hline & Fun. & Beh. & Str. & Fun. & Beh. & Str. & Fun. & Beh. & Str. & Fun. & Beh. & Str. \\
\hline Tablet & 1 & 27 & 0 & 0 & 3 & 1 & 0 & 0 & 5 & 0 & 0 & 0 \\
\hline Rhino with & 6 & 66 & 11 & 0 & 0 & 0 & 0 & 85 & 0 & 0 & 0 & 0 \\
\hline MS & (6) & (66) & (11 & & & & & (85) & & & & \\
\hline Word/Pow & & & ) & & & & & & & & & \\
\hline erPoint & & & & & & & & & & & & \\
\hline (MS & & & & & & & & & & & & \\
\hline Word/Pow & & & & & & & & & & & & \\
\hline erPoint & & & & & & & & & & & & \\
\hline representat & & & & & & & & & & & & \\
\hline ion is & & & & & & & & & & & & \\
\hline given in & & & & & & & & & & & & \\
\hline bracket) & & & & & & & & & & & & \\
\hline Notes- & 11 & 27 & 9 & 0 & 0 & 42 & 4 & 28 & 4 & 0 & 0 & 0 \\
\hline taker & & & & & & & & & & & & \\
\hline
\end{tabular}

The influences of the design tools on the textual representation of functional, behavioural and structural preliminary ideas is statistically insignificant $\left(F_{\text {textual_functional }}(2,5)=0.95, p\right.$ $\left.=0.448 ; F_{\text {textual_behavioural }}(2,5)=1.60, p=0.29 ; F_{\text {textual_structure }}(2,5)=0.22, p=0.812\right)$. With all the three design tools, there is no graphical function and behaviour representation of preliminary ideas. Also in the eighteen experiments conducted, in only two experiments did the designer minimally uses the tool to the represent the graphical structure of preliminary ideas. Observations from Table 8 are the following: 
- The results in Table 4 indicate that the number of preliminary concepts is higher in Rhino with MS Word/PowerPoint and the Notes-taker tools. With reference to this observation, it is surprising to note that Rhino along with word processing software such as MS Word or Power Point provides more descriptions of preliminary concepts both in the original and redesign experiments. This could be due to the designer's perception that there may be limitations in expressing the design intent. This is because even in describing fewer concepts, the designers had to put in a lot of effort to describe these concepts in Rhino.

- As noted in the case of requirements, graphical elements are used to represent mainly the structural elements of preliminary concepts in the original experiments with the Notes-taker tool.

Table 9 shows the textual and graphical content in terms of the functional, behavioural and structural elements of the captured detailed concepts in the original and redesign experiments.

Table 9. Representation formats of the captured detailed concepts in the original and redesign experiments (Function/Behaviour/Structure) 


\begin{tabular}{|c|c|c|c|c|c|c|c|c|c|c|c|c|}
\hline \multirow[t]{3}{*}{ Tools } & \multicolumn{6}{|c|}{ Original } & \multicolumn{6}{|c|}{ Redesign } \\
\hline & \multicolumn{3}{|c|}{ Textual } & \multicolumn{3}{|c|}{ Graphical } & \multicolumn{3}{|c|}{ Textual } & \multicolumn{3}{|c|}{ Graphical } \\
\hline & Fun. & Beh. & \begin{tabular}{|l} 
Str. \\
\end{tabular} & Fun. & Beh. & Str. & Fun. & Beh. & \begin{tabular}{|l} 
Str. \\
\end{tabular} & Fun. & Beh. & Str. \\
\hline Tablet & 6 & 60 & 70 & 27 & 4 & 101 & 18 & 86 & 32 & 0 & 3 & 70 \\
\hline Rhino with & 9 & 137 & 34 & 0 & 0 & 29 & 0 & 114 & 38 & 0 & $1(0)$ & 14 \\
\hline MS & (9) & $(137$ & (34 & & & (4) & & (114) & $(38$ & & & (0) \\
\hline Word/Pow & & ) & ) & & & & & & ) & & & \\
\hline erPoint & & & & & & & & & & & & \\
\hline (MS & & & & & & & & & & & & \\
\hline Word/Pow & & & & & & & & & & & & \\
\hline erPoint & & & & & & & & & & & & \\
\hline representat & & & & & & & & & & & & \\
\hline ion is & & & & & & & & & & & & \\
\hline given in & & & & & & & & & & & & \\
\hline bracket) & & & & & & & & & & & & \\
\hline Notes- & 39 & 310 & \begin{tabular}{|l|l}
193 \\
\end{tabular} & 0 & 8 & 117 & 5 & 77 & 50 & 0 & 0 & 56 \\
\hline taker & & & & & & & & & & & & \\
\hline
\end{tabular}

The influence of the design tools on the textual representation of functional, behavioural and structural detailed concepts is statistically insignificant $\left(F_{\text {textual_functional }}(2,5)=0.75, p\right.$ $\left.=0.520 ; \mathrm{F}_{\text {textual_behavioural }}(2,5)=2.40, \mathrm{p}=0.186 ; \mathrm{F}_{\text {textual_structure }}(2,5)=0.59, \mathrm{p}=0.588\right) . \mathrm{In}$ eighteen experiments, the function of the detailed concept is categorized only once, and the behaviour of the detailed concepts is categorized only in six design experiments in graphical representation. Hence, the statistical significance is not identified with these parameters. The influence of the design tools on the graphical representation of structural detailed concepts is statistically significant $\left(F_{\text {graphical_structure }}(2,5)=13.06, p=0.010\right)$. 
Figure 8 describes the mean plot for the graphical representation of the structure of detailed concepts with reference to design tools, design problems and designers. Figure 8 points out that the number of graphical components to illustrate the structure of detailed concepts is very low with Rhino with MS Word/PowerPoint tool.

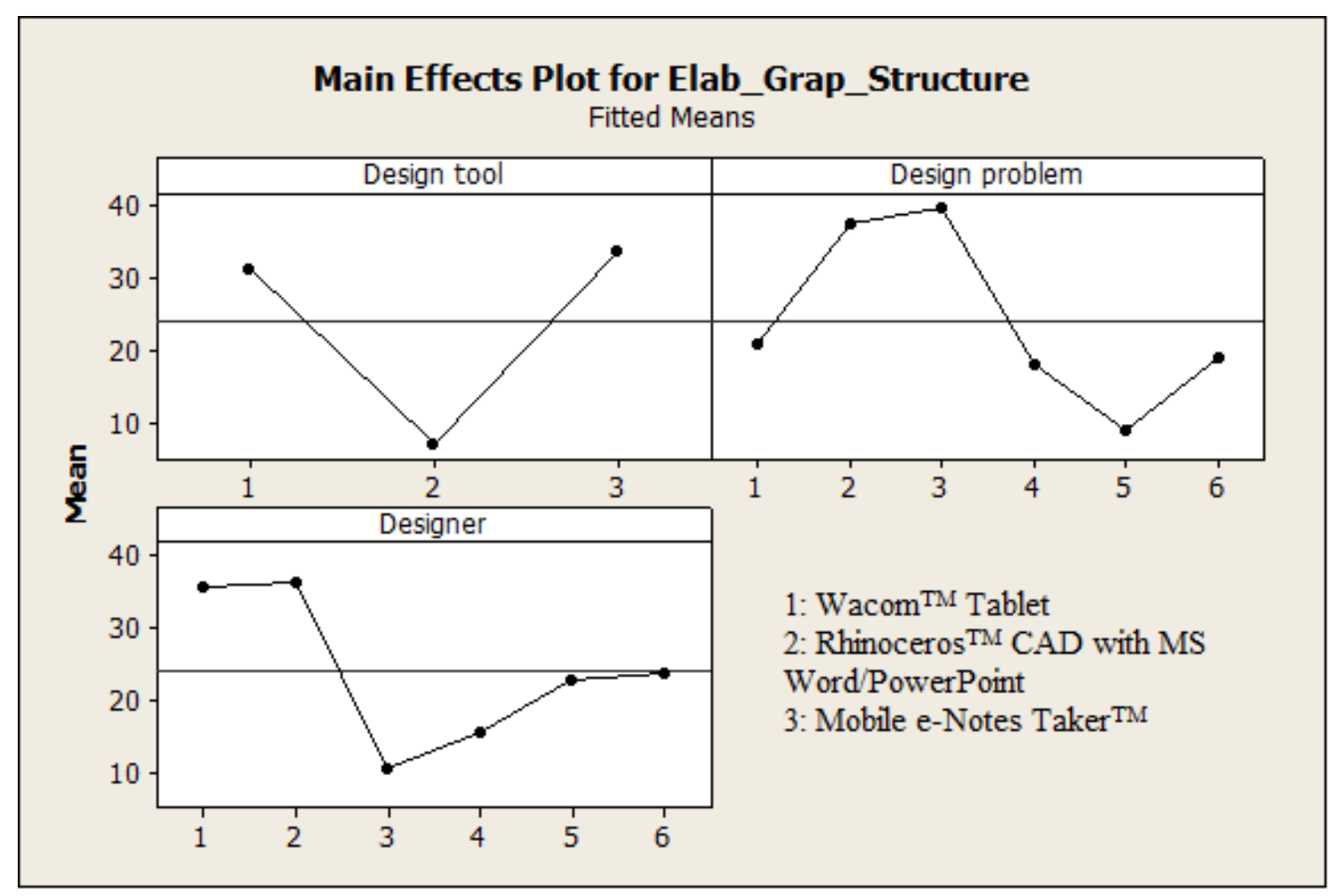

Figure 8. The mean plot for the Graphical Representation of the Structure of Detailed Concepts with reference to design tools, design problems and designers

The observations from Table 9 are the following:

- The Tablet is the only tool in which functions are graphically represented in the original experiments. Figure 9 illustrates this statement through the graphical diagrams drawn by a designer. 

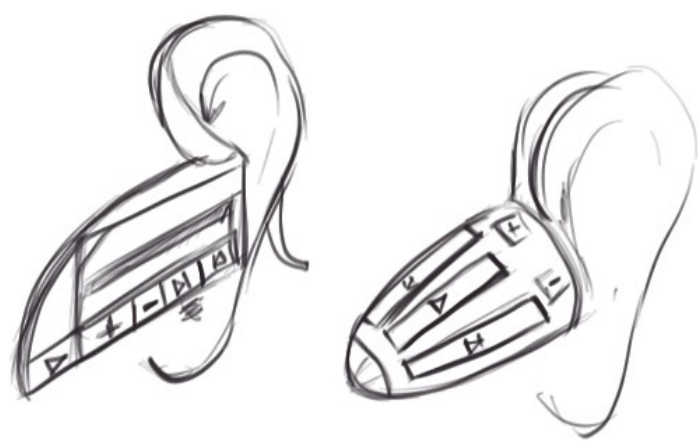

Figure 9. Illustration of graphical function representation (play, volume and skip functions)

- Both in the original and redesign experiments, graphical structural components are fewer in Rhino with MS Word/PowerPoint compared to those in the other two tools. Since only a few distinguishable structural components with precision are captured in Rhino with MS Word/PowerPoint, the factors mentioned by Robertson et al. (2007) such as large amount of detail and interconnectedness and the complexity of the model influencing premature fixation might be questionable. Also, the fact that fewer distinguishable components were not captured could be due to the designers finding it difficult to express them and felt this might clash with thinking.

- They are more textual descriptions of function, behaviour and structural elements with the Notes-taker tool in the original experiments. However, in the redesign experiments, the textual descriptions in all the elements are substantially reduced.

- Comparing Table 9 with reference to Figure 4 reveals that even with a lower proportion of $58 \%$ of time consumed in the elaboration and detailing of concepts in the original experiments with the Notes-taker, the number of distinguished elements captured is substantial. But with $72 \%$ of time consumed in the same stages with Rhino with MS Word/PowerPoint, there are fewer distinguished elements. From this observation, it should be noted that the more time taken to 
represent concepts does not necessarily have a higher impact on the wider representations of function, behaviour and structure elements. The level of precision necessary in articulating the concepts could be a reason for the resistance to change and a wide representation with Rhino with MS Word/PowerPoint. In other words, only precision in representation (especially structure elements) is increased with more time spent, possibly at the cost of completeness.

The observations indicate that conceptual design tools have a significant impact on the textual representation of structural requirements, and the graphical representation of the structural elements of detailed concepts. Also, the influence of the designer on the textual representation of behavioural requirements is statistically significant. The amount of time taken to capture each concept does have an impact on the representation of the captured concepts, but in varying levels of precision and expression elements.

H5. The formats of the representation of captured documents have a significant impact on reinterpretation in the redesign phase.

Two parameters are used to test this hypothesis. These are: (i) the percentage of time spent on the reinterpretation of all the original design documents and (ii) the issues incurred during the reinterpretation process. Table 10 compares the percentage of time spent in the reinterpretation of the entire original document with the total number of concepts and the percentage of statements captured with reference to the designer's articulated statements in the original design experiments. The percentage values of the statements captured in the original experiments with reference to designer articulated statements are taken from Annamalai Vasantha and Chakrabarti (2010). Except with Rhino with MS Word/PowerPoint, the amount of time spent by the designer in the reinterpretation of the original captured documents is substantial in the redesign experiments. However, comparing Tables $7-9$ reveals that the reinterpretation time spent 
with the Notes-taker could be lower in relation to the amount of content captured textually. In Rhino with MS Word/PowerPoint, the time spent in reinterpretation could be less due to the significantly lower percentage of statements captured in the original experiments.

Table 10. Percentage of time spent in the reinterpretation of captured original documents in the redesign experiments

\begin{tabular}{|c|c|c|c|c|}
\hline \multirow[t]{2}{*}{ Tools } & \multirow{2}{*}{$\begin{array}{l}\text { Percentage of } \\
\text { time spent in } \\
\text { reinterpretation } \\
\text { of all the original } \\
\text { documents }\end{array}$} & \multicolumn{2}{|c|}{$\begin{array}{c}\text { Total number of } \\
\text { concepts }\end{array}$} & \multirow[b]{2}{*}{$\begin{array}{l}\text { Percentage of } \\
\text { statements } \\
\text { captured in } \\
\text { original design } \\
\text { documents with } \\
\text { reference to } \\
\text { designer } \\
\text { articulated } \\
\text { statements } \\
\text { (Annamalai } \\
\text { Vasantha and } \\
\text { Chakrabarti, 2010) }\end{array}$} \\
\hline & & Preliminary & Detail & \\
\hline Tablet & 31 & 2 & 10 & 35.8 \\
\hline $\begin{array}{c}\text { Rhino with MS } \\
\text { Word/PowerPoint }\end{array}$ & 17 & 12 & 5 & 23.4 \\
\hline Notes-taker & 34 & 14 & 8 & 40.5 \\
\hline
\end{tabular}


In order to identify issues with the reinterpretation process, video protocols were analysed to find the problems mentioned and the symptoms expressed by the re-designers. The original documents were thoroughly used by the designers in all the redesign experiments, irrespective of the tools used. The designers spent considerable time in understanding the work of the previous designers. Table 11 lists and groups issues in the categories of understanding, ambiguity, assumptions and repetition. Observations from Table 11 are the following:

- There are more issues related to the understanding of original designs than for ambiguity, assumption and repetition issues.

- Understanding the original designer's intention from drawings is a big issue noted while using the Tablet.

- Issue related to 'didn't understand fully the previous designer's concept' is observed across all the tools. Most of the time, these kinds of concepts were ignored during redesign.

- Natural handwriting as an input provided by the Tablet and the Notes-taker created problems in reinterpretation due to poor writing and sketches.

- The improper labelling of concepts seems to be a problem during reinterpretation across all the tools. There are a few times when the re-designers understood the concept with the title alone. This designer-behaviour shows that the designers were interested in understanding only the overall working principle of the concepts, rather than looking into the details of the concepts.

- Comparing Tables 9 and 11 shows that the use of more elements to describe the detailed concepts in the original documents through the Notes-taker does not lead to more issues during reinterpretation in redesign experiments. 
With these observations and issues identified, we concluded that the formats of the representation of captured concepts have a significant impact on reinterpretation in the redesign phase.

Table 11. Issues identified during reinterpretation of original documents in redesign experiments

\begin{tabular}{|c|c|c|c|c|}
\hline \multirow[t]{2}{*}{ Issues with } & \multirow[t]{2}{*}{ Aspects } & \multicolumn{3}{|c|}{ Number of instances } \\
\hline & & Tablet & $\begin{array}{c}\text { Rhino with } \\
\text { MS } \\
\text { Word/Power } \\
\text { Point }\end{array}$ & $\begin{array}{l}\text { Notes- } \\
\text { taker }\end{array}$ \\
\hline \multirow[t]{10}{*}{ Understandin } & $\begin{array}{c}\text { Designer's intention from } \\
\text { drawing }\end{array}$ & 11 & 3 & 0 \\
\hline & $\begin{array}{l}\text { Technical aspect of the } \\
\text { concepts from drawing }\end{array}$ & 1 & 2 & 0 \\
\hline & $\begin{array}{l}\text { Didn't understand fully the } \\
\text { previous designer's concept }\end{array}$ & 6 & 4 & 6 \\
\hline & Poor hand writing & 5 & $\mathbf{0}$ & 3 \\
\hline & Poor sketch & 0 & $\begin{array}{c}1 \text { (used MS } \\
\text { Word) }\end{array}$ & 3 \\
\hline & Terminology used & 2 & 1 & 0 \\
\hline & Not labelled the concept & 2 & 3 & 2 \\
\hline & Rationale of evaluation value & 1 & 1 & 0 \\
\hline & $\begin{array}{l}\text { Evaluation criteria due to } \\
\text { poor labelling }\end{array}$ & 1 & 0 & 1 \\
\hline & Requirement & 0 & 1 & 4 \\
\hline
\end{tabular}




\begin{tabular}{|c|c|c|c|c|}
\hline & Sub-total & 29 & 16 & 19 \\
\hline \multirow[t]{3}{*}{ Ambiguity } & $\begin{array}{l}\text { Choosing previous concept } \\
\text { due to representation of tick } \\
\text { mark in all the concepts }\end{array}$ & 1 & 0 & 0 \\
\hline & About behaviour of a concept & 0 & 0 & 1 \\
\hline & Sub-total & 1 & $\mathbf{0}$ & 1 \\
\hline \multirow[t]{5}{*}{ Assumption } & $\begin{array}{l}\text { About a task which might } \\
\text { have been done or not done } \\
\text { by the original designer }\end{array}$ & 1 & 2 & 0 \\
\hline & Behaviour of a concept & 1 & 0 & 0 \\
\hline & Evaluation method used & 1 & 0 & 0 \\
\hline & $\begin{array}{l}\text { Similarity with the previous } \\
\text { concept }\end{array}$ & 1 & 0 & 0 \\
\hline & Sub-total & 4 & 2 & $\mathbf{0}$ \\
\hline \multirow[t]{4}{*}{ Repetition } & $\begin{array}{l}\text { Derived the same function } \\
\text { done by the previous } \\
\text { designer }\end{array}$ & 1 & 1 & 0 \\
\hline & $\begin{array}{l}\text { Redraw previous sketch for } \\
\text { better understanding }\end{array}$ & 1 & 0 & 1 \\
\hline & Sub-total & 2 & 1 & 1 \\
\hline & Overall total & 36 & 19 & 21 \\
\hline
\end{tabular}

H6. Designer adaptability to a design tool has a significant impact on the representation and reinterpretation of captured documents. 
To verify this hypothesis, video protocols are analysed to note explicit mention of a designer's comfort and discomfort during interaction with the design tools, both during original and redesign experiments. Observations from these analyses are listed below. Using the Tablet,

- One designer requested for a mouse input device along with the Tablet pen.

- One designer mentioned that he liked the tablet and said that it helped to design sketches faster and better.

- One designer could not modify the previous file because the layers used were inaccessible due to the storage format.

- One designer spent about two minutes for precisely drawing a single line and expressed that it was difficult to draw a line.

Using Rhino with MS Word/PowerPoint,

- One designer said that he could do conceptual designing better on paper.

Using the Notes-taker,

- One designer expressed unhappiness about his sketches made using this tool.

- One designer had problems with the pen interface because he had to apply pressure to draw.

Other tool-features such as image resolution, working area size and tool inclination are not mentioned as a problem by any of the designers. The aforementioned statements indicate that the designers are conscious about the tools they use during the conceptual design. However, only a few issues highlighted sporadically by the designers show that the designers had become accustomed to the conceptual design tools in terms of the ability and usability of the tools. Adaptability is not found to be an issue with the assessed tools. 
Therefore, we argue that the representation and reinterpretation of the captured concepts might not have been influenced by designer adaptability to these design tools; all the designers became quickly accustomed to the tools so that there was no significant variability in adaptation visible across tools.

\section{Discussion}

Figure 10 summarizes the statistically significant research findings in the influence diagram from analyses of the experimental results. In this globalised competitive design environment, designers are increasingly being pressured to create quality innovative products in faster cycles. In this stressful environment, choosing the right tool could have a strong influence on the amount of time spent by the designers to solve the given problem without influencing the design outcomes (e.g. the number of preliminary and detailed design concepts). The variation of time spent is largely accounted for in the detailed design activity. As the design tools do not statistically influence the number of preliminary and detailed design concepts, more in-depth investigations are required to understand the influences of product, process and environment. The statistical influences of design tools on the textual representation of structural requirements and the graphical representation of the structure of detailed concepts show that the designers have to understand these behavioural changes while using different tools, because it can subsequently have an impact on the reinterpretation process during the redesign phase. The results show that the generated redesign concepts are fewer, both in the preliminary and detailed, compared to those in the original experiments; this is irrespective of the design tool used. The potential causes for this designer-behaviour include the following:

- The percentage of the capture of the designer's articulation $(23.4 \%-40.5 \%$ in the original experiments) is substantially low with all the design tools. The capture of 
the designer's articulation is the ratio of the 'designer's captured statements in design documents to the 'designer's articulated (expressed explicitly) statements'.

- Even though each designer went thoroughly through the original design documents, due to this low capture of the designer's intention in the original document, the designer only acquired the overall working principle of the concepts rather than their details.

- A fixation with the original concepts is often noted in the experiments. This could be due to the redesigners being overly impressed by the original concepts.

- The redesign itself seems to be a restricted process due to the problems identified in the originally chosen concept being the only ones addressed. The designers do not take the opportunity of considering the extensive solution search space available in the original design.

- Also, many original concepts are ignored during redesign due to the lack of or partial understanding of the concepts. The ambiguity of the concepts is also a factor.

- Low motivation on the part of the re-designer could be another reason for the reduced time spent and the fewer concepts generated in the redesign process. 


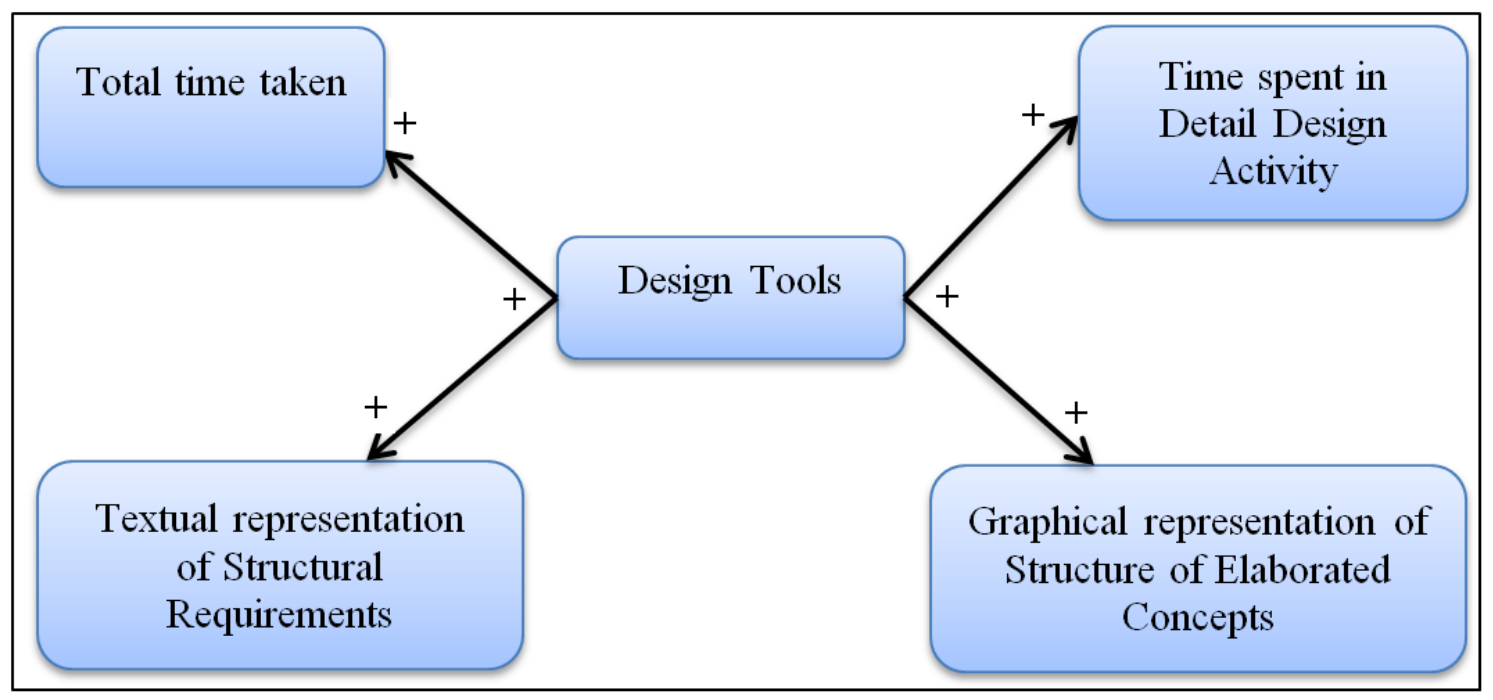

Figure 10. The influence diagram from the statistically significant research findings

In the literature, ambiguity is said to facilitate lateral transformations to generate alternative ideas. But the analyses of the results from our experiments suggest that novice re-designers often ignored the concepts that were difficult and ambiguous to understand. This observation lends support to the argument that designers might require training for reinterpretation skills for extracting the necessary information from the concepts originally captured, rather than working with poor understanding, ambiguity and assumptions about the original designer's intent. Also, design tools need to aid the reinterpretation process; none of the tools currently support the capture of all of the designer's articulations required for the redesign process.

Even though we did not aim to find an equivalent problem in each set, the statistical analysis did not represent the influence of the problem on the studied variables. The influence of the designers is statistically observed in the total time taken, time spent on the requirement generation activity, and the textual representation of behavioural requirements.

After visualizing the structural elements from Rhino, one designer stressed the need for a technical description of the concepts. To facilitate this description, notable proposals such as the representation of the functional properties of design objects to accommodate 
multiple views of design objects in a collaborative CAD environment (Roseman and Gero, 1996) and agent models (Maher et al. 2007) to monitor and augment the designer in capturing and reusing required information might need to be explored for supporting conceptual paper-based and CAD tools. But to build effective agent models to support reinterpretation, the core descriptive research question to be answered is 'Which designer's articulations are not captured but should be otherwise during the design process'. As pointed out by Stacey and Eckert (2003), the answer to this question should cover three aspects of a design: what - the design itself; how - by what procedure the artefact should be generated; and why - the reason why the design should be as it is.

Another issue is about the graphical and textual representation of the captured content. McKoy et al. (2001) argue that graphical information proves more useful for idea generation than textual information. But our experimental results and analyses reveal issues in both graphical and textual information during the redesign process, for all the three tools used. Poor hand writing while using the Notes-taker and the Tablet leads to misunderstanding, whereas graphical diagrams are not rich enough to understand the original designer's intention. It should be noted that the student designers' (lack of) skills in tool use could have influenced the (lack of) richness of concept representation. Simplicity, ease of use and ease of learning of the tool might have played a vital role in the concept generation process.

Bonnardel and Zenasni (2010) argue that technology developments should be adapted to the designers' cognitive processes instead of requiring them to adapt to new technologies. However, considering the highly adaptable nature of the designers, it is difficult to find the real cognitive, technological needs of the designers. In addition to the think aloud and video protocol, different observations and analyses are required to obtain an understanding of the designer's cognitive tool needs. 
The foremost implication from these results is that there is a need to help designers understand and learn the facilities provided by design tools and their influences on the design process. From the industrial perspective, the efficacy of design tools in capturing and reusing concepts in appropriate representations for better reinterpretation during the redesign process needs to be established.

\section{Conclusions and Future Work}

This paper focuses on understanding the influence of conceptual design tools: Mobile eNotes Taker ${ }^{\mathrm{TM}}$, Wacom ${ }^{\circledR}$ Tablet, and Rhinoceros ${ }^{\circledR}$ CAD with MS Word/PowerPoint on concept representation and reinterpretation, during original as well as redesign phases. Compared to many studies cited in the literature which adopted a limited sample size, our work used a symmetrical set of design of experiments with three different design problems framed for original and another three for redesign experiments. Six designers solved individually three different problems using three different tools, where each time a different problem was solved using a different tool. Overall, eighteen experiments were analysed to test the hypotheses framed. Recorded videos, protocol transcripts and captured documents provided a rich array of data for the analyses.

The analyses reveal the areas where the analysed tools need better mechanisms to support designers in original and redesign processes. Critical needs for increasing the number and time to be spent on generating preliminary concepts, enriching textual and graphical representation of detailed concepts, and eliminating issues in the reinterpretation process are highlighted as major findings. An influence diagram constructed from statistically significant results highlights the definite impact of design tools on four critical design parameters: the total time taken, time spent on detailed design activity, the textual representation of the structural requirements and the graphical representation of the structure of detailed concepts. 
It should be noted that the three tools evaluated in detail were evaluated only when the designers worked individually. This needs to be extended for all design stages, for design teams, and for both collaborative (where designers work together in the same place and time) and distributed (where designers work together in different places and at the same time) set-ups. Also, these results are produced from using novice designers as subjects/participants. It is vital to test the framed hypotheses with expert designers and compare the results. It should be noted that all these results are influenced by the variable amount of time taken by each designer to solve the given problem. Forcing designers to work compulsorily for one hour might have changed their behaviour and significantly influenced the results obtained. Another set of experiments is required to compare and contrast the normal and forced duration experiments. Also it should be noted that the designers' competence before each experiment was not judged and the experiments were started after getting permission from them. Their competence level could have influenced the results. The influence of the competence level will be carried out in the further research. More descriptive studies are required to advance understanding in the representation and reinterpretation processes for developing better design tools for designers to support the development of innovative solutions.

\section{References}

Annamalai Vasantha, G.V. and Chakrabarti, A., 2010. Assessment of Design Tools for Knowledge Capture and Reuse. International Conference on Modelling \& Management of Engineering Processes, University of Cambridge, UK.

Bilda, Z. and Gero, J. S., 2005. Do We Need CAD during Conceptual Design? Martens, B., and Brown, A., (eds.), Computer Aided Architectural Design Futures, 155164 , Springer.

Bilda, Z. and Demirkan,H., 2003. An insight on designers' sketchin activities in traditional versus digital media. Design Studies, 24, 27-50. 
Black, A., 1990. Visible planning on paper and on screen: the impact of working medium on decision-making by novice graphic designers. Behaviour \& Information Technology, 9, 283-296.

Blessing, L. Chakrabarti, A. and Wallace K., 1995. A design research methodology. Proc. Int. Conf. Engineering design, 502-507.

Bonnardel, N. and Zenasni, F., 2010. The impact of technology on creativity in design: An enhancement? Creativity and innovation management, 19(2), 180-191.

Chakrabarti, A. Sarkar, P. and Leelavathamma, N. B., 2005. A functional representation for aiding biomimetic and artificial inspiration of new ideas, Artificial Intelligence for Engineering Design, Analysis and Manufacturing, 19, 113-132.

Chakrabarti, A., 2001a. Sharing in Design: Categories, Importance and Issues, Proc. of the Intl. Conference on Engineering Design (ICED01), Design Methods for Performance and Sustainability, 563-570, Glasgow.

Chakrabarti, A., 2004. A new approach to structure sharing, ASME Journal of Computing and Information Science in Engineering, 4(1), 11-19.

Chakrabarti, A., 2001b. Improving efficiency of procedures for compositional synthesis by using bidirectional search, Artificial Intelligence for Engineering Design, Analysis and Manufacturing, 15(1), 67-80.

Cham, J. G. and Yang, M. C., 2005. Does sketching skill relate to good design? In Proceedings ASME 2005 International Design Engineering Technical Conferences \& Computers and Information in Engineering Conference, Long Beach, California, ASME, Philadelphia.

De Bono, E., 1970. Lateral thinking: Creativity step by step. NY, US: Harper \& Row.

Elsen, C., Darses, F., and Leclercq, P., 2010. An Anthropo-based Standpoint on Mediating Objects: Evolution and Extension of Industrial Design Practices. Design Computing and Cognition DCC'10, Gero, J.S., (ed), Springer.

Fish, J. and Scrivener, S., 1990. Amplifying the minds eye: sketching and visual cognition. Leonardo, 23, 117-126.

Goel, V., 1995. Sketches of thought. US: MIT.

Goldschmidt, G., 1994. On visual design thinking: the vis kids of architecture. Design Studies, 15, 158-174.

Hannah, R. Joshi, S. and Summers, J.D., 2012. A user study of interpretability of engineering design representations, Journal of Engineering Design, 23(6), 443468. 
Hewson, R., 1994. Marking \& making: a characterisation of sketching for typographic design, $\mathrm{PhD}$ Thesis, Open University, UK.

Ibrahim, R. and Rahimian F. P., 2010. Comparison of CAD and manual sketching tools for teaching architectural design. Automation in Construction, 19, 978-987.

Ibrahim, R. and Paulson Jr, B.C., 2008. Discontinuity in organisations: identifying business environments affecting efficiency of knowledge flows in PLM. Intl. J. Prod. Lifecycle Manage, 3, 21-36.

Jonson, B., 2005. Design ideation: the conceptual sketch in the digital age. Design Studies, 26(6), 613-624.

Kelly, T., 2001. The art of innovation: Lessons in creativity from IDEO, America's leading design firm. New York: Currency/Doubleday.

Kwon, J. Choi, H. Lee, J. and Chai, Y., 2005. Free-Hand Stroke Based NURBS Surface for Sketching and Deforming 3D Contents. PCM 2005, Part I, LNCS 3767.

Lawson, B., 1997. How designers think: The design process demystified. Oxford, UK, Architectural Press.

Lawson, B., 2002. CAD and Creativity: Does the Computer Really Help? Leonardo, $35(3), 327-331$.

Levet F. Granier, X. and Schlick, C. 2006. 3D sketching with profile curves, LNCS 4073, $114-125$.

Liu, Y-C. Chakrabarti, A. and Bligh, T.P., 2000. A Computational Framework for Concept Generation and Evaluation in Mechanical Design: Further Developments of FuncSION, Artificial Intelligence in Design'00, J.S. Gero (Ed.), 499-519, Kluwer Academic Publishers.

Maher, M. L. Rosenman, M. and Merrick, K., 2007. Agents for multidisciplinary design in virtual worlds. Artificial Intelligence for Engineering Design, Analysis and Manufacturing, 21, 267-277.

McKoy, F.L., et al., 2001. Influence of design representation on effectiveness of idea generation. In Proceedings of ASME 2001 international design engineering technical conference and computers and information in engineering conference, Pittsburgh, PA. Paper no D ${ }^{\mathrm{TM}}$-DETC 2001-21685. 1-10.

Nagai, Y. and Taura, T., 2006. Formal description of Concept Synthesizing Process for Creative Design, Design Computing and Cognition (DCC'06), 443-460, Springer, Netherlands. 
Rahimian P. F. Ibrahim, R. and Jaafar, F.Z., 2008. Feasibility study on developing 3D sketching in virtual reality (VR) environment. Int. J. Susta. Trop. Des. Res. Pract. $3,60-78$.

Renzulli, J. S. Owen, S. V. and Callahan, C. M., 1974. Fluency, flexibility, and originality as a function of group size. The Journal of Creative Behavior, 8(2), 107-113.

Robertson, B.F. Walther, J. and Radcliffe, D.F., 2007. Creativity and the use of CAD tools: Lessons for engineering design education from industry. Journal of Mechanical Design, July, 129, 753-760.

Robertson, B.F. and Radcliffe, D.F., 2009. Impact of CAD tools on creative problem solving in engineering design. Computer-Aided Design, 41, 136-146.

Rosenman, M. A. and Gero, J. S., 1996. Modelling multiple views of design objects in a collaborative CAD environment. Computer-Aided Design, 28(3), 193-205.

Sarkar, P. and Chakrabarti, A., 2011. Assessing design creativity. Design Studies, 32, 348-383.

Sarkar, P. and Chakrabarti, A., 2007. Understanding Search in Design, International Conference on Engineering Design (ICED07), Paris, France.

Sarkar, P. and Chakrabarti, A., 2008. The effect of representation of triggers on design outcomes, Artificial Intelligence for Engineering Design, Analysis and Manufacturing, 22(2), 101-116.

Sarkar, P. Phaneendra, S. and Chakrabarti, A., 2008. Developing engineering products using inspiration from nature, ASME Journal of Computing and Information Science in Engineering, 8, 2008.

Sartori, J. Pal, U. and Chakrabarti, A., 2010. A Methodology for Supporting 'Transfer' in Biomimetic Design, Amaresh Chakrabarti and Li Shu (Eds.), Artificial Intelligence for Engineering Design, Analysis and Manufacturing, Vol.24, No.4, 483-505, 2010.

Schön, D., 1983. The reflective practitioner: How professionals think in action. Surry England: Ashgate Publishing Limited.

Srinivasan, V. and Chakrabarti, A., 2010a. Investigating Novelty-Outcome Relationship in Engineering Design. Artificial Intelligence for Engineering Design, Analysis and Manufacturing, 24, 161-178.

Srinivasan, V. and Chakrabarti, A., 2010b. An Integrated Model of Designing, Special Issue on Knowledge Based Design. Ashok K. Goel and Andrés Gómez de Silva 
Garza (eds.), ASME Journal of Computing and Information Science in Engineering, 10(3).

Stacey, M. and Eckert, C., 2003. Against ambiguity. Computer-Supported Cooperative Work, 12, 153-183.

Stones, C. M. and Cassidy, T., 2007. Comparing synthesis strategies of novice graphic designers using digital and traditional design tools. Design Studies, 28, 59-72.

Stones, C. and Cassidy, T., 2010. Seeing and discovering: how do student designers reinterpret sketches and digital marks during graphic design ideation? Design Studies, 31, 439-460.

Whitefield, A., 1986. An Analysis and Comparison of Knowledge Use in Designing with and without CAD, in Smith A., (ed.) Proceedings of CAD, Butterworth, London.

Won, P. H., 2001. The comparison between visual thinking using computer and conventional media in the concept generation stages of design. Automation in Construction, 10, 319-325.

\section{List of Appendixes:}

Appendix 1. Tools features - $\underline{\mathrm{http}: / / \mathrm{goo} . \mathrm{gl} / 0 \mathrm{C} 6 \mathrm{Bsh}}$

Appendix 2. Design problems - http://goo.gl/Nq63v

Appendix 3. Parameter definition - http://goo.gl/wjxLP

Appendix 4. Data sets and results from Minitab ${ }^{\mathrm{TM}}$ software - http://goo.gl/tIggW8 


\section{List of figures:}

Figure 1. Conceptual design tools used in study: (a) - (c)

Figure 2. Research hypotheses map

Figure 3. An example of preliminary and detailed concepts generated for laptop security issues

Figure 4. Distribution of time spent on design activities in original experiments (all nine combined)

Figure 5. Distribution of time spent on design activities in redesign experiments (all nine combined)

Figure 6. Mean plot for total time with reference to design tool, design problem and designer

Figure 7. Illustration of the textual and graphical classification into function, behaviour and structure elements

Figure 8. The mean plot for the Graphical Representation of the Structure of Detailed Concepts with reference to design tools, design problems and designers

Figure 9. Illustration of graphical function representation (play, volume and skip functions)

Figure 10. The influence diagram from the statistically significant research findings 


\section{List of tables:}

Table 1. Features of design tools

Table 2. Structure of the design of experiments (D1 - D6: Designers, P1 - P3: Original problem; P1' - P3': Redesign problem) (crossed experiments were not conducted due to experimental constraints)

Table 3. Details about the subjects who participated

Table 4. Number of preliminary concepts generated in the original and redesign experiments

Table 5. Number of detailed concepts generated in the original and redesign experiments

Table 6. Total time taken by the designers in original and redesign experiments (in minutes)

Table 7. Representation formats of captured requirements combining all original and redesign experiments (Function/Behaviour/Structure)

Table 8. Representation formats of the captured preliminary concepts in the original and redesign experiments (Function/Behaviour/Structure)

Table 9. Representation formats of the captured detailed concepts in the original and redesign experiments (Function/Behaviour/Structure)

Table 10. Percentage of time spent in the reinterpretation of captured original documents in the redesign experiments

Table 11. Issues identified during reinterpretation of original documents in redesign experiments 